\title{
Cooperative Overlay Spectrum Access in Cognitive Radio Networks
}

\author{
Wei Liang ${ }^{1}$, Soon Xin $\mathrm{Ng}^{2}$ and Lajos Hanzo ${ }^{2}$ \\ ${ }^{1}$ School of Computing and Communications, Lancaster University, LA1 4WA, UK. \\ ${ }^{2}$ School of ECS, University of Southampton, SO17 1BJ, UK. \\ Email: ${ }^{1}$ w.liang@lancaster.ac.uk; ${ }^{2}\{$ sxn,lh\}@ecs.soton.ac.uk, http://www-mobile.ecs.soton.ac.uk
}

\begin{abstract}
In order to mitigate the shortage of wireless spectrum, the appealing concepts of cooperative communication techniques and cognitive radio networks have been combined for the sake of improving the spectral efficiency and hence the overall system throughput. We mainly survey the overlay spectrum access scheme in this novel cooperative cognitive radio (CCR) network context. Therefore the interference between the Licensed Users/Primary Users (PUs) and the Unlicensed Users/Cognitive Users (CUs) can be offset by relying on some of the CUs to act as Relay Nodes (RN). More specifically, we have investigated the cooperative relaying technique in the context of the overlay spectrum access scheme aiming for allowing the PUs to transmit at a lower power and/or at a higher throughput, while at the same time enabling the CUs to communicate using the bandwidth released. Additionally, gaming techniques can be employed for negotiating between the PUs and the CUs for determining the specific fraction of relaying and active transmission time. Therefore, we will consider two main schemes in the overlay spectrum access scheme based on the CCR network, which are the frequency division based channel as well as the timedivision based channel. Moreover, we have surveyed the relevant advances concerning the game-based model of the overlay-based CR network. Specifically, both the family of non-cooperative and cooperative games as well as matching games have been reviewed. Furthermore, we will review the joint design of coding, modulation, user-cooperation and CCR techniques, which leads to significant mutual benefits for both the PUs and CUs.
\end{abstract}

Index Terms-Cognitive Radio network, Cooperative Communication, Dynamic Network Coding, Game theory, Overlay Spectrum Access scheme, Adaptive Coded Modulation.

\section{NOMENCLATURE}

$\begin{array}{ll}\text { AAF } & \text { Amplify and Forward } \\ \text { BPS } & \text { Bit Per Symbol } \\ \text { CR } & \text { Cognitive Radio } \\ \text { CU } & \text { Cognitive User } \\ \text { CCR } & \text { Cooperative Cognitive Radio } \\ \text { CDA } & \text { Conventional Distributed Algorithm } \\ \text { DN } & \text { Destination Node } \\ \text { DAF } & \text { Decoder and Forward } \\ \text { MABC } & \text { Multiple Access Broadcast Channel } \\ \text { OWR } & \text { One Way Relay } \\ \text { PU } & \text { Primary User } \\ \text { PDA } & \text { Pragmatic Distributed Algorithm } \\ \text { RN } & \text { Relay Node } \\ \text { SN } & \text { Source Node } \\ \text { SAS } & \text { Spectrum Access Scheme } \\ \text { TWR } & \text { Two Way Relay } \\ \text { TDBC } & \text { Time Division Broadcast Channel }\end{array}$

The financial support of the European Research Council's Advanced Fellow Grant, that of the India-UK Advanced Technology Center, as well as that of the European Union's Seventh Framework Program (FP7/2007-2013) under the auspices of the CONERTO project (grant agreement no 288502) is gratefully acknowledged.

\section{INTRODUCTION}

Since the turn of the century, Cognitive Radio (CR) and cooperative communication techniques have been extensively considered in the literature for the sake of efficiently improving the exploitation of the wireless radio resources. In order to solve the spectrum shortage problem, the following two aspects have been investigated [1]:

- Exploration of hitherto unused spectrum, as in mm-wave [2] visible light [3] and Terahertz communications [4].

- Identifying and opportunistically exploiting the spectrum holes [5] that are momentarily unused by the licensed owners of the spectrum.

This paper aims for investigating a combination of CR techniques with cooperative communication schemes for improving the exploitation of the spectrum. We commence by reviewing the corresponding literature and standard, before the novel solutions are proposed.

In [6], Mitola and Maguire stated that "radio etiquette is the set of RF bands, air interfaces, protocols, and spatialtemporal patterns that moderate the use of radio spectrum. CR extends the software radio with radio-domain model-based reasoning about such etiquette." In wireless communications, CR constitutes a design paradigm for a network or a wireless node, which could change its transmission mode efficiently in order to communicate by avoiding the interference with the licensed user/ Primary User (PU) or the unlicensed user/ Cognitive User (CU). Goldsmith et al. [7] stated that in the terminology of information theory, the CR is a wireless communication system that intelligently utilizes any available side information about the a) channel conditions, b) codebooks, c) activity, and d) message of other nodes with which it shares the spectrum. Specifically, a CR is a specific type of spectrum sensing assisted cooperative scheme, where the cooperation efficiency critically depends on the amount of knowledge exchange between the CUs and PUs [7]. In Haykin's paper [8], it was stated that a CR constitutes a highly reliable communications device ensuring that the radio spectrum can be efficient exploited. The radio spectrum is a precious and scarce resource. Numerous wireless communication engineers have made efforts to maximize the exploitation of the radio spectrum. Interestingly, in November 2002, the Federal Communications Commission (FCC) demonstrated that the actual licensed spectrum is largely unoccupied most of the time [9] and hence they planned to reshape the traditional models of spectral allocation and control. Clearly, the static 
spectrum allocation has resulted in low-efficiency exploitation of the precious spectral resources. Another recent measurement shows that the average spectrum occupancy in the band spanning from $30 \mathrm{MHz}$ to $3 \mathrm{GHz}$ over six cities is $5.2 \%$ and that the maximum total spectrum occupancy is $13.1 \%$ in New York City [10]. In order to resolve the contradiction between the static the spectrum allocation, and its low real exploitation, opportunistic access of the under-utilized licensed frequency bands has been proposed [10]. Additionally, the CR technique allows users to utilize and share the available spectrum, which is not fully ${ }^{1}$ occupied in either space, or in time or in fact in the joint space-time domain, in an opportunistic manner. CUs are allowed to detect the available spectrum, adjust to detect the PUs present in the spectrum and to coordinate with other CUs. In the CR terminology, PUs have a higher priority or 'legacy rights' for the usage of a specific part of the spectrum. By contrast, the CUs have a lower priority and they should exploit the spectrum without causing interference to PUs. Hence the CUs have to have CR capabilities, including the sensing of the spectrum that has not been occupied by the PUs and may exploit the unused spectrum in order to improve its exploitation. Specifically, an overview of spectrum sensing techniques has been provided in [5], [11]. Moreover, a CR is capable using or sharing the spectrum in an opportunistic manner, with the aid of a spectrum sharing technique. The family of spectrum sharing techniques enable the CUs to coordinate their access with the primary channel [12]. In [13], the Medium Access Control (MAC) protocol of cognitive ad hoc networks has been investigated by Jia et al., which makes informed sensing decisions after exploring all the spectral access opportunities. This process is different from the classic physical layer issue of how to detect the existence of primary user signals and then exploits these opportunities for the secondary users' transmissions. Li et al. [14] conceived a sophisticated spectrum-sensing consensus based scheme, where the population of agents maintains coordination based on local interactions without centralized information exchange. Additionally, Yu et al. [15] proposed a novel biologically inspired consensus-based cooperative spectrum sensing scheme for CR networks. A comprehensive list of major security threats occuring within a CR network has been surveyed by Attar et al. [16]. Based on these insightful contributions it may be inferred that the CR technology has a significant impact on the upper layer performance of wireless networks, particularly in mobile ad hoc networks. As a further contribution, Guan et al. [17] discussed the topology control and routing issues of CR networks. Against this background, in this article, we focus our attention on the associated spectrum sharing issues.

Cooperative communication [18] relies on the broadcast nature of wireless communications in order to allow the nodes to help each other for the sake of attaining the same advantages as those offered by Multiple-Input Multiple-Output (MIMO) systems. As a benefit, they are capable of improving the attractive communication capacity and transmission integrity. Various cooperative techniques have been widely investigated since the turn of the century. In [19], [20], several classical

\footnotetext{
${ }^{1}$ The term 'fully' is with respect to the 'radio-temperature' or 'capacity achievable'.
}

cooperative protocols were evaluated in terms of their power saving, diversity order and outage probability. The diversitymultiplexing trade-off was quantified in [21]. Specifically, relay selection, optimal resource allocation, as well as networkcoded cooperation have been investigated in [22]-[24]. More explicitly, various half-duplex two-phase cooperative techniques were proposed for recovering the $50 \%$ throughput loss experienced by conventional One-Way Relaying (OWR) scheme [25], such as Two-Way Relaying (TWR) [26] and successive relaying [27] systems.

In a nutshell, $\mathrm{CR}$ is a novel technology that can potentially improve the exploitation of the radio spectrum and cooperative communications plays a key role in the development of CR networks. The applications of cooperative communication approaches in the context of CR networks have been discussed in [1], [28]-[35], [35]-[42]. Cooperative transmission can greatly improve the spectrum access opportunity as well as sharing efficiency for CUs with the help of cooperative RN. Cooperative relaying is widely regarded as the key technology in CR networks [43]. The applications of cooperative relaying in CR systems have also been discussed in [28][31]. At the time of writing research efforts are invested in determining the optimum power allocation and in simplifying the relay selection process in cooperative CRs [34]-[36]. Relay selection techniques have been employed in multiple-relay $\mathrm{CR}$ networks with the aim of improving the performance of the second-hop transmission [32], [33]. The main consideration in relay selection and power allocation in CR networks are related to improving the overall spectral efficiency and to the reduction of the interference [34]-[36]. Additionally, various resource allocation techniques have been conceived for CRaided wireless networks over the space-, time- and frequencydomain for improving the attractive spectral efficiency [37][39]. Furthermore, diverse spectrum sharing protocols have been combined with TWR in CR networks, where two PUs communicate with each other with the assistance of the CUs acting as the relay [35], [40], [41].

Therefore, cooperative communication aided CR systems may be categorized into the following three types:

- Cooperation among the PUs;

- Cooperation between PUs and CUs [43]-[46];

- Cooperation among the CU peers [47], [48].

More specifically, the first type is similar to the traditional cooperative communication, while in the third type, a $\mathrm{CU}$ may act as a Relay Node (RN) for other CUs, which may have different available spectra [47]. For the second type, the PUs have a higher priority than the CUs, where the CUs may act as RN for the PUs [44]. Another interesting protocol involving simultaneous transmissions of the PUs and CUs has been proposed in [43] for maximizing the overall achievable rate. In this paper, we commence by reviewing the advances in spectrum sharing of CR networks, specifically focusing on cooperative communications. Cooperative transmission can greatly improve the spectrum access opportunity as well as sharing efficiency for CUs with the help of cooperative RN. More explicitly, cooperative communication inspired a range of new design concepts capable of dramatically improving the 


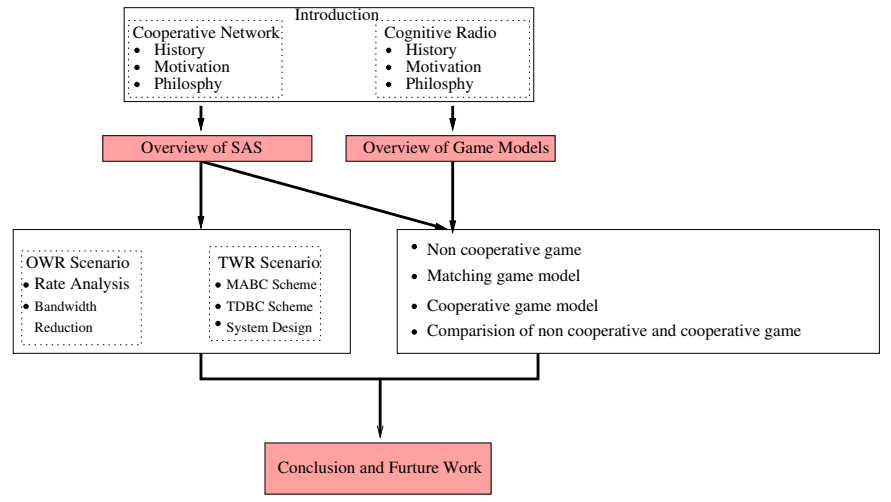

Fig. 1. The outline of this paper.

spectral efficiency of wireless networks.

As mentioned in Section I, the combination of cooperative communication and CR networks has been developed for the sake of mitigating the spectrum shortage as well as improving the spectral efficiency in the existing spectral band. Hence cooperative CR networks were advocated in this treatise and the average throughput achieved by Adaptive Trellis Turbo Coded Modulation (ATTCM) [49] was investigated. The outline of this paper is presented in Fig. 1. Our goal is to stimulate further research and to inspire additional novel contributions on spectral-efficient CR networks. In Section II, we have reviewed the three basic types of CR based spectrum access schemes, followed by a brief introduction to game-theoretic models. The family of relaying channel models has been considered in Section V, including both one-way and two-way relaying systems. Furthermore, a cooperative game-theoretic model has been proposed for an overlay spectrum sharing CR scheme, as detailed in Section VI. Finally, we have concluded in Section VII. The list of symbols shown in this paper has been presented in Table I.

\begin{tabular}{|l||l|}
\hline$T$ & The time slot length \\
\hline$W$ & The Bandwidth \\
\hline$\sigma$ & The channel variance \\
\hline$L$ & The number of PUs \\
\hline$K$ & The number of CUs \\
\hline$l$ & The index of PUs \\
\hline$k$ & The index of CUs \\
\hline$R_{l, r e q}^{P U}$ & The rate requirement of $l$ th PU \\
\hline$R_{k, \text { req }}^{C U}$ & The rate requirement of $k$ th $\mathrm{CU}$ \\
\hline$\beta_{l, k}$ & The time allocation factor between $l$ th PU and $k$ th $\mathrm{CU}$ \\
\hline$\tau$ & The step size of time allocation fraction \\
\hline$\epsilon_{l, k}$ & The time fraction between $\mathrm{Pt}_{l}$ and $\mathrm{Ct}_{k} / \mathrm{RN}$ \\
\hline$\alpha$ & The path-loss exponent \\
\hline
\end{tabular}

TABLE I

THE LIST OF SYMBOLS.

\section{OVERVIEW OF SPECTRUM SHARING SCHEMES}

A range of spectrum sensing solution have been investigated by various researchers [11], [50]-[54], [54]. The existing contributions in spectrum sharing may be classified according to three salient aspects [55], namely the architecture, the spectrum allocation regime and the spectrum access technique, as shown in Fig. 2. The architecture can be classified either as centralized or as a distributed scheme. In a centralized scheme, the spectrum allocation and access procedures are controlled by a central controller or entity. All users or nodes send their information to the central controller. Then a spectrum allocation map is constructed by the central controller, which has the authority to lease spectrum to users/ nodes in a limited geographical region for a specific amount of time [56]-[58]. By contrast, the spectrum allocation and access is typically based on local information, which is gleaned by each distributive user/node in the distributed scheme [59], [60]. Moreover, several recent contributions [61]-[63] reveal that distributed solutions tend to closely follow the centralized philosophy, but have a lower complexity. More particularly, one of the main contributions of [61] is the development of an appealingly low-complexity distributed algorithm, which is capable of approaching the performance of the centralized solution. Additionally, in [64], the authors have proved that the computational complexity of the distributed algorithm is low than the centralized algorithm.

Additionally, an optimal centralized scheme based on a game-theoretic model has been discussed in [65], where the proposed centralized solution was not based on exhaustive search - instead the objective function was optimized with the aid of a mathematical tool box.

Moreover, the spectrum allocation regime of Fig. 2 can be classified into non-cooperative and cooperative schemes. Noncooperative spectrum sharing [66], [67] typically results in a reduced spectrum efficiency regime, but does not require frequent message exchanges. By contrast, in the cooperative spectrum sharing, a common technique is to form a cluster to share the users' information locally [68]. The cooperative approaches tend to outperform the non-cooperative approaches and result in a certain fairness, as well as an improved throughput. On the other hand, the non-cooperative approach imposes a lower information exchange requirement and hence requires less energy [69]. Furthermore, there are three main

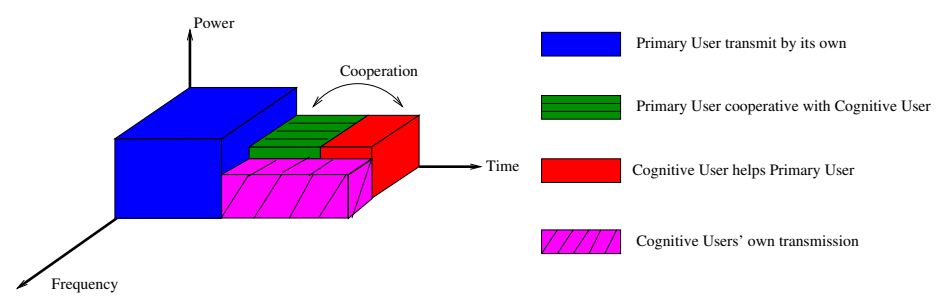

Fig. 3. Overlay spectrum model of CR network.

paradigms conceived for spectrum access in CR networks [7]:

- Underlay spectrum sharing scheme (SAS) [7]: CUs can transmit simultaneously with PUs by using the same frequency spectrum, under the constraint that the interference inflicted by the CUs on the PUs does not degrade the PU's communication quality. In this scheme, the CUs are not required to perform spectrum sensing. However, the interference caused by the CUs' transmission must not exceed the tolerable threshold.

- Overlay SAS [7]: CUs can transmit simultaneously with 


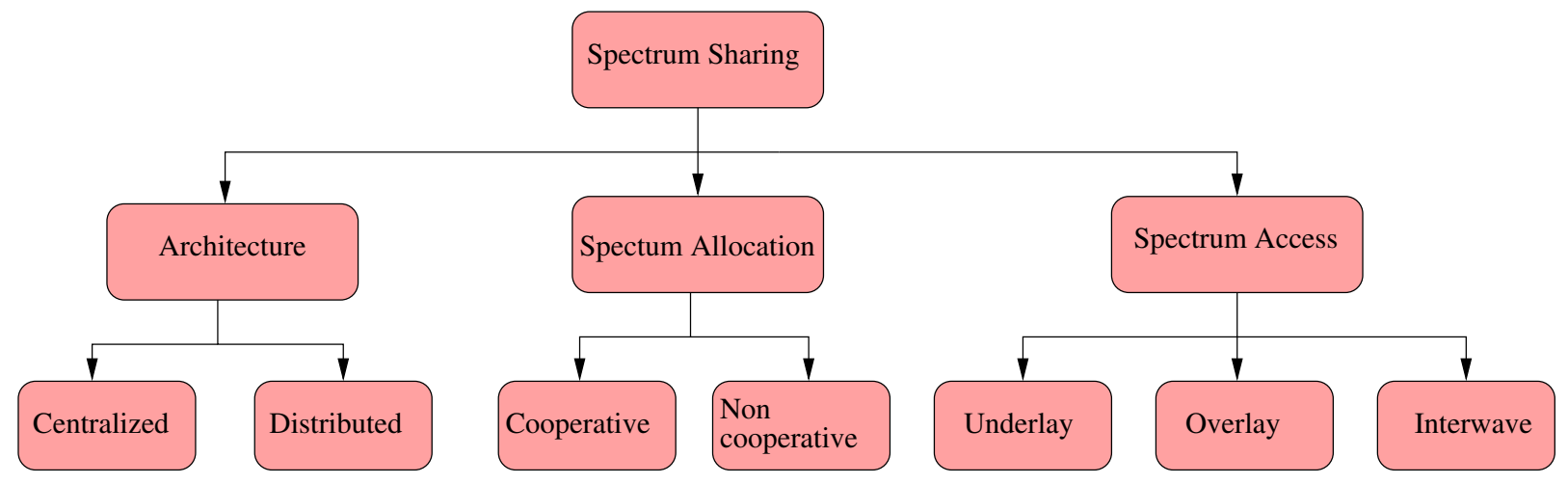

Fig. 2. The classification of spectrum sharing in CR network.

PUs in the same frequency slot. The knowledge sharing and cooperation between the CUs and PUs is critical in the overlay model. Specifically, the interference imposed on the PUs can be offset by using part of the CUs' power for relaying the PUs' information. As shown in Fig. 3, some CUs assist the PUs to free up some spectrum bands. These vacant spectrum bands would then be used by other CUs for their secondary transmission.

- Interwave SAS [7]: The CUs would only transmit simultaneously with the PUs, when a busy spectral slot was wrongly detected as a spectral hole. Specifically, the CUs exploit the spectrum slots, which are not utilized most of the time for their secondary communication. Hence the spectrum efficiency is improved.

In the interwave technique, the knowledge sharing between the PU and CU is critical for ensuring that the CUs' transmissions do not interfere with those of the PUs. The CUs opportunistically communicate over the spectrum hole in order to minimize the interference imposed on the PUs. The underlay and overlay paradigms permit the concurrent communications of the PUs and CUs. By contrast, the main goal of the interwave paradigm is to avoid the simultaneous transmission of the PUs and CUs. Moreover, the underlay system requires accurate knowledge of the interference imposed by the CUs' transmitter on the PUs' receiver. By contrast, the overlay scheme needs a large amount of side-information, such as the non-causal knowledge of the PU's codebook. Furthermore, the interweave regime also requires considerable side-information about the PUs or the existing users and this information can be obtained from PUs' spectrum sensing action. The distributed cooperation aided overlay and underlay paradigm of CR networks has been discussed in [70], where in the overlay system the CUs were allowed to opportunistically access the radio spectrum allocated to the PUs, provided that the relays offered relaying services for the PUs. Therefore, the knowledge of sharing and cooperation between the CUs and PUs in the overlay SAS have been literature in [71], [72]. In contrast to the overlay scheme, in the underlay scheme, a CU distributively selects the frequency of the channel and the transmission power level for maximizing its level of satisfaction while at the same time avoiding any excessive interference imposed on the PUs.

Although these three schemes rely on distinct approaches, their advantages can be combined by constructing to hybrid schemes. For example, the underlay and overlay schemes are combined in [73], [74], where the CUs invoke the spectrum overlay technique, if spectrum holes are found. Otherwise, the spectrum underlay technique will be employed. Additionally, the authors of [75] introduced stochastic resource allocation algorithms for both the interweave and underlay paradigms, where the CUs can access the frequency band only if no $\mathrm{PU}$ is active in the context of the interweave and overlay paradigm. By contrast, in the underlay paradigm CUs can access the channel even when the PUs are active, provided that they adjust their transmission power so that the interference imposed on the active PUs remains below a specified threshold [76]. In our work, we have mainly considered the overlay scheme. The significant difference between the overlay scheme and the interwave regime is that the cooperation of the PUs and CUs is actively supported by the overlay scheme. In the overlay scheme of [77], the PU's performance is enhanced by exploiting the benefit of cooperative diversity with the aid of the CU acting as a RN, while CU's transmission is carefully coordinated by the PU's transmission scheduling. A "win-win" scenario has been constructed for both PU and CU which gives both the PU and CU an incentive to cooperative. Therefore, the overlay scheme constitutes an opportunistic spectrum access scheme, as discussed in [76]. A summary of underlay SAS and overlay SAS are shown in Table II.

\section{OverView OF GAME MOdel}

Game theory is one of the techniques that can be beneficially used for spectrum sharing in CR networks as described in [8]. To elaborate further, a game is defined by a set of players, a set of actions for each player and the payoffs for the players [90]. A player chooses an action and the associated complete plan of action is referred as the strategy. However, most of the game theory models rely on the equilibrium concept, which ensures that a player could gain either a fair or an optimal pay-off under a given strategy of the other players [91][93]. More specifically, a strategy is deemed to have reached equilibrium, when it becomes impossible to reward a specific player without disadvantaging other players [90]. Explicitly, in Nash equilibrium, no player has any intention to change its strategy to gain a higher payoff, provided that the other players also maintain their current strategies. The definition of Nash 


\begin{tabular}{|c|c|c|}
\hline Schemes & Author(s) & Contribution \\
\hline \multirow{6}{*}{ Underlay Spectrum Access Scheme } & Laneman et al. [78] & $\begin{array}{l}\text { The underlying techniques exploit space diversity available through cooperating } \\
\text { terminals' relaying signals for multi-path propagation in wireless networks. }\end{array}$ \\
\hline & Kehao Wang et al. [79] & $\begin{array}{l}\text { An underlay CR communication system in which a CU can access multiple primary } \\
\text { spectrum channels only when its interference to the PU is limited. }\end{array}$ \\
\hline & Khoshkholgh et al. [80] & $\begin{array}{l}\text { An interference management method for underlay spectrum sharing are proposed, } \\
\text { which the secondary service has been facilitated by granting passive access to the } \\
\text { power control signalling transmitted by the primary network base station. }\end{array}$ \\
\hline & GaoJie et al. [81] & $\begin{array}{l}\text { A decode-and-forward buffer-aided relay selection has been proposed for underlay } \\
\text { cognitive relay networks in the presence of both primary transmitter and receiver. }\end{array}$ \\
\hline & Benitez Olivo et al. [82] & Underlay scheme employed for multi-user cognitive radio network. \\
\hline & Rasti et al. [83] & $\begin{array}{l}\text { A distributed power control algorithm to address the uplink interference manage- } \\
\text { ment problem in CR networks where the underlaying CUs share the same licensed } \\
\text { spectrum with the PUs in multi-cell environments. }\end{array}$ \\
\hline \multirow{6}{*}{ Overlay Spectrum Access Scheme } & Huang et al. [84] & $\begin{array}{l}\text { Spectrum sharing between wireless networks improves the efficiency of spectrum } \\
\text { usage, and thereby alleviates spectrum scarcity due to growing demands for wireless } \\
\text { broadband access. }\end{array}$ \\
\hline & Gür et al. [85] & $\begin{array}{l}\text { The conventional femtocell idea with an infrastructure-based overlay cognitive } \\
\text { network paradigm has been combined in a femtocell-based CR architecture for } \\
\text { enabling multitiered opportunistic access in next-generation broadband wireless } \\
\text { systems. }\end{array}$ \\
\hline & Lin et al. [86] & $\begin{array}{l}\text { The overlay orthogonal spectrum between device-to-device and enhanced cellular } \\
\text { networks. }\end{array}$ \\
\hline & Kwon et al. [87] & $\begin{array}{l}\text { Investigates efficient spectrum sharing methods for single-input-multiple-output } \\
\text { networks where nodes with different capabilities and requirements are spatially } \\
\text { distributed according to homogeneous Poisson point processes. }\end{array}$ \\
\hline & El-Sherif et al. [88] & $\begin{array}{l}\text { The joint design of routing and resource allocation algorithms in cognitive radio } \\
\text { based wireless mesh networks. }\end{array}$ \\
\hline & Kim et al. [89] & An improved spectrum-sharing protocol for multiuser cooperation in CR networks. \\
\hline
\end{tabular}

TABLE II

SUMMARY OF UNDERLAY AND OVERLAY SASS.

Equilibrium is as follows: Let $L$ be the number of players in a game, where the user-index is $l$, which obeys $0<l \leq L$, while $s_{l}$ denotes a set of potentially available mixed strategies for player $l$, with $s_{l} \in \mathcal{S}_{i}$ being any possible strategy of player 1. The Nash Equilibrium satisfies the following equation:

$$
\pi_{l}\left(s_{l}^{\prime}, s_{L-l}^{\prime}\right) \geq \pi_{l}\left(s_{l}, s_{L-l}^{\prime}\right)
$$

where $\pi_{l}$ represents the payoff function of player $l, s_{l}^{\prime}$ represents the Nash Equilibrium strategy of player $l$, while $s_{L-l}^{\prime}$ constitute the Nash Equilibrium strategies of all players other than player $l$. Game theoretic techniques can be divided into two types, namely non-cooperative and cooperative games. In a non-cooperative game, the players make their decisions independently and aim for maximizing their own utility. By contrast, the players in the cooperative game cooperate with each other for maximizing their total utility.

Some game theoretic models that have been employed in CR networks are summarized in Table III. A fair resource allocation method is proposed based on the Nash Bargaining solution for a problem, where a group of CUs access the resources of a primary system [108]. PUs and CUs form a coalitional game, where they can pay charges to each other to motivate the cooperation [63]. A stackelberg-game was employed for controlling the user's behaviour by broadcasting the relevant information in heterogeneous cognitive networks [102]. Users are given an incentive to share the spectrum in a cooperative way. To enforce user cooperation, defecting users may be asked to pay a tax [98]. A double auction mode is invoked for analyzing the interaction among the Wireless Regional Area Network (WRAN) service provides, TV broadcasters and WRAN users [94].
A repeated game can be seen as a static $^{2}$ non-cooperative strategic game that is repeated over time. By repeating a game many times, the players (users) may become aware of their past behaviors and change their strategies accordingly. In the repeated game context, all players are better off, if they cooperate. More specifically, if a game is played repeatedly, then the mutually desired outcome can be reached, where each player believes that a defection for short-term personal gain will terminate the cooperation, hence resulting in a subsequent loss for the player that outweighs the potential short-term gain [90]. The threat of future 'punishments' prevents any player acting rationally from defection. In [112], a repeated game among the PUs' transceivers was formulated to show that the collusion can indeed be maintained, provided that all the PUs are aware of the potential punishment. However, if a primary service deviates from the collusion, then all the other primary services will resort to the punishment action permanently. In this case, the primary services will consider the long-term benefits for themselves. The cooperation among the users would avoid a fact, which users competing for the open spectrum may have no incentive to cooperative with each other, and they many even exchange false private information about their channel conditions in order to get more access to the spectrum [98]. Moreover, power control strategies have been considered for the CUs in the repeated game proposed in [99], [113]. Specifically, in [99], the CUs are capable of controlling the power by observing the interference imposed by them on the PUs upon exploiting both the feedback signals

\footnotetext{
${ }^{2} \mathrm{~A}$ game is static, if the players carry out their actions only once and independent by each other [90]. Specifically, a static game is a one-shot game, where all players make decisions without any knowledge of the strategies chosen by the other players [91].
} 


\begin{tabular}{|c|c|c|c|}
\hline Game Type & Model & Author(s) & Contribution \\
\hline \multirow{12}{*}{ Non-cooperative gam } & \multirow{4}{*}{ Auction game } & Niyato et al. [94] & $\begin{array}{l}\text { A double auction mode is invoked for analyzing } \\
\text { the interaction among the Wireless Regional } \\
\text { Area Network (WRAN) service provides, TV } \\
\text { broadcasters and WRAN users. }\end{array}$ \\
\hline & & Huang et al. [95] & $\begin{array}{l}\text { The SNR auction and the power auction were } \\
\text { proposed for determining relay selection and } \\
\text { relay power allocation in a distributed fashion. }\end{array}$ \\
\hline & & Wang et al. [96] & $\begin{array}{l}\text { A bandwidth auction problem is considered, in } \\
\text { which each CU makes a bid for the amount of } \\
\text { spectrum and each PU may assign the spectrum } \\
\text { among the CUs by itself according to the infor- } \\
\text { mation from the CUs without degrading its own } \\
\text { performance. }\end{array}$ \\
\hline & & Wang et al. [97] & $\begin{array}{l}\text { A distributed relay selection mechanism based } \\
\text { on a Vickrey auction game is proposed for the } \\
\text { uplink user cooperation multiple access wireless } \\
\text { networks. }\end{array}$ \\
\hline & \multirow{4}{*}{ Repeated game } & Wu et al. [98] & $\begin{array}{l}\text { Users are given an incentive to share the spec- } \\
\text { trum in a cooperative way. To enforce user } \\
\text { cooperation, defecting users may be asked to pay } \\
\text { a tax. }\end{array}$ \\
\hline & & Zhou et al. [99] & $\begin{array}{l}\text { Reinforcement Learning for Repeated Power } \\
\text { Control Game in Cognitive Radio Networks. }\end{array}$ \\
\hline & & Xiao et al. [100] & $\begin{array}{l}\text { By using intervention in repeated games we } \\
\text { can achieve a larger set of equilibrium payoffs } \\
\text { and loosen requirements for users' patience to } \\
\text { achieve a target payoff. }\end{array}$ \\
\hline & & Hamouda et al. [101] & $\begin{array}{l}\text { A cooperative physical resource blocks (PRBs) } \\
\text { sharing scheme in a dual-hop LTE-Advanced } \\
\text { in which every node can transmit on some of } \\
\text { the PRBs assigned to a coexisting node without } \\
\text { causing it harmful interference. }\end{array}$ \\
\hline & \multirow{4}{*}{ Stackelberg game } & Haddad et al. [102] & $\begin{array}{l}\text { A stackelberg-game was employed for control- } \\
\text { ling the user's behaviour by broadcasting the } \\
\text { relevant information in heterogeneous cognitive } \\
\text { networks. }\end{array}$ \\
\hline & & Nan et al. [103] & $\begin{array}{l}\text { Stackelberg Game for Bandwidth Allocation } \\
\text { in Cloud-Based Wireless Live-Streaming Social } \\
\text { Networks. }\end{array}$ \\
\hline & & Zhu et al. [104] & $\begin{array}{l}\text { Joint Mode Selection and Spectrum Partitioning } \\
\text { for Device-to-Device Communication. }\end{array}$ \\
\hline & & Yi et al. [105] & $\begin{array}{l}\text { The dynamic spectrum access among multiple } \\
\text { heterogeneous primary spectrum owners and } \\
\text { CUs in recall-based cognitive radio networks is } \\
\text { investigated. }\end{array}$ \\
\hline \multirow{7}{*}{ Cooperative game } & \multirow{3}{*}{ Coalitional game } & Li et al. [63] & $\begin{array}{l}\text { PUs and CUs form a coalitional game, where } \\
\text { they can pay charges to each other to motivate } \\
\text { the cooperation. }\end{array}$ \\
\hline & & Cheung et al. [106] & $\begin{array}{l}\text { Investigate a distributed MAC protocol using the } \\
\text { more accurate signal-to-interference-plus-noise- } \\
\text { ratio model in CR network. }\end{array}$ \\
\hline & & $\begin{array}{l}\text { Mochaourab } \\
\text { al. }[107]\end{array}$ & $\begin{array}{l}\text { The cooperation between the links using coali- } \\
\text { tional games where the links in a coalition either } \\
\text { perform zero forcing transmission or Wiener } \\
\text { filter precoding to each other. }\end{array}$ \\
\hline & \multirow{4}{*}{ Bargaining game } & Attar et al. [108] & $\begin{array}{l}\text { A fair resource allocation method is proposed } \\
\text { based on the Nash Bargaining solution for a } \\
\text { problem, where a group of CUs access the } \\
\text { resources of a primary system. }\end{array}$ \\
\hline & & Zhang et al. [109] & $\begin{array}{l}\text { Wireless cooperative communications require } \\
\text { appropriate spectrum allocation (SA) and power } \\
\text { allocation (PA) between the source and relay } \\
\text { nodes. }\end{array}$ \\
\hline & & Liu et al. [110] & $\begin{array}{l}\text { The coordination and bargaining between two } \\
\text { selfish users over a Gaussian interference chan- } \\
\text { nel. }\end{array}$ \\
\hline & & Zhang et al. [111] & $\begin{array}{l}\text { The joint uplink subchannel and power allo- } \\
\text { cation problem in cognitive small cells using } \\
\text { cooperative Nash bargaining game theory has } \\
\text { been considered. }\end{array}$ \\
\hline
\end{tabular}

TABLE III

SUMMARY OF GAME MODE USED IN CR NETWORKS. 
of the PUs and the knowledge of the transmission rates obtained during the previous step.

\section{Adaptive Trellis Coded Modulation}

As early as 1968, Hayes demonstrated that an efficient technique of mitigating the detrimental effects of channel fading is to adaptively adjust the modulation and/or the channel coding format as well as a range of other system parameters based on the near-instantaneous channel quality information perceived by the receiver, which is fed back to the transmitter with the aid of a feedback channel [49]. In 1996 Torrance and Hanzo [114] proposed a set of mode switching levels designed for achieving a high average BPS throughput, while maintaining a specific average BER. As a further developement in 1997, Chua and Goldsmith invoked channel coding in conjunction with adaptive modulation in a narrow-band environment [115]. In an effort to provide a fair comparison of the various coded modulation schemes, $\mathrm{Ng}$, Wong and Hanzo [116] have found that TTCM was the best scheme at a given decoding complexity in the coded modulation family of Trellis Coded Modulation (TCM), TTCM, Bit-Interleaved Coded Modulation (BICM) and Iterative-Decoding assisted BICM (BICM-ID). Hence we focused our attention on this power- and bandwidth-efficient TTCM scheme in this paper. More details on the TTCM principles may be found in [117]. The research contributions on Adaptive Coded Modulation (ACM) emerging during 1968 to 2006 have been studied in [118]. The milestones disseminated in the literature after 2010 are shown in Table. IV. The near-instantaneous Adaptive TTCM modes are controlled by the near-instantaneous channel conditions. More specifically, a more vulnerable, but higherthroughput TTCM mode, such as TTCM based 32QAM or 64QAM can be employed, when the channel conditions are good, while a lower-throughput but more robust TTCM mode is used, namely TTCM aided 4PSK, when the channel conditions are poor. More specifically, ATTCM is capable of maximizing the throughput, when the channel quality improves and vice versa, whilst meeting the target-BER requirements.

\section{A. System Structure}

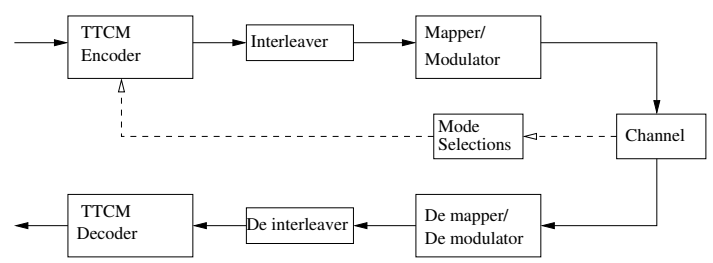

Fig. 4. The schematic of ATTCM scheme (c) [114].

The schematic of the near-instantaneous ATTCM arrangement is depicted in Fig. 4. The transmitter extracts the ATTCM mode signalled back by the receiver employing a sophisticated mode selection mechanism in order to adjust the ATTCM mode according to the prevalent channel condition. The nearinstantaneously adaptive scheme requires a reliable feedback link from the receiver to the transmitter. The effective throughput (or iBPS ) of the ATTCM encoder modes is given by:
- No transmission (NoTx): 0 iBPS;

- TTCM-QPSK(or 4PSK): 1 iBPS;

- TTCM-8PSK: 2 iBPS;

- TTCM-16QAM: 3 iBPS;

- TTCM-32QAM: 4 iBPS;

- TTCM-64QAM: 5 iBPS;

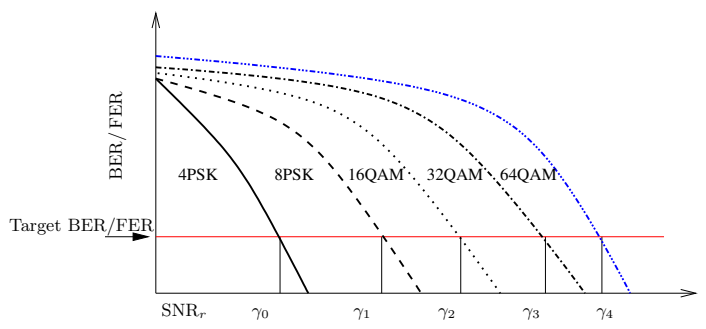

Fig. 5. Mode selection according to a target BER or FER.

1) Mode-Switching Operation of ATTCM: The ATTCM mode switching thresholds $\Upsilon=\left[\gamma_{0}, \gamma_{1}, \gamma_{2}, \gamma_{3}, \gamma_{4}\right]$ are determined based on the required target BER or FER performance curves of each of the five TTCM schemes, as shown in Fig. 5. Based on the target BER or FER, the related mode-switching thresholds can be obtained. Specifically, the ATTCM mode switching operation is based on the following algorithm:

$$
\text { MODE }= \begin{cases}\gamma_{R}>\gamma_{4}, & \text { TTCM-64QAM; } \\ \gamma_{3}<\gamma_{R} \leq \gamma_{2}, & \text { TTCM-32QAM; } \\ \gamma_{2}<\gamma_{R} \leq \gamma_{3}, & \text { TTCM-16QAM; } \\ \gamma_{1}<\gamma_{R} \leq \gamma_{2}, & \text { TTCM-8PSK; } \\ \gamma_{0}<\gamma_{R} \leq \gamma_{1}, & \text { TTCM-4PSK; } \\ \gamma_{R} \leq \gamma_{0}, & \text { No-Tx; }\end{cases}
$$

where $\gamma_{R}$ is the SNR at the receiver. Hence, an appropriate TTCM modulation mode can be selected according to the instantaneous received SNR $\gamma_{R}$ using Eq. (2).

\section{CoOperative Relay techniques FOR OVERlay SAS}

In this section, the implementation of relay techniques for overlay SAS will be discussed. The overlay SAS is illustrated in Fig. 3. Explicitly, in overlay SAS, the CU acts as the RN by relaying the PU's signal in exchange for gaining access to some of the frequency band or to a faction of the PU's TS in order to carry out their own secondary transmission. Therefore, we have two types of schemes, as shown in Fig. 6, which are based on the classic time-division as well as followed by its frequency-division principles. We will employ the frequencydivision philosophy in this section, followed by its timedivision counterpart.

\section{A. Frequency-division channel model}

To facilitate efficient spectrum sharing between the PU and CUs, we consider configuring and sharing the frequency bands of $W_{1}$ and $W_{2}$, as shown for the frequency-division channel of Fig. 6. Observe for the frequency-division channel of Fig. 6 that the CUs act as the RNs and assist the PU/SN in transmitting its signal in one of the frequency bands, seen 


\begin{tabular}{|c|l|l|}
\hline Year & Author(s) & Contribution \\
\hline \hline 2010 & Djordjevic [119] & Adaptive modulation and coding for Free-Space Optical channels. \\
\hline 2011 & Jiang [120] & $\begin{array}{l}\text { A singular-value-based adaptive modulation and cooperation scheme for virtual- } \\
\text { MIMO systems. } \\
\text { Simulating LTE cellular systems: an open-source framework. }\end{array}$ \\
\hline 2012 & Li et al. [122] & $\begin{array}{l}\text { Scalable video multicast with adaptive modulation and coding in broadband wireless } \\
\text { data systems. }\end{array}$ \\
\hline 2013 & Mastronarde [123] & $\begin{array}{l}\text { Joint physical layer and system level power management for delay sensitive wireless } \\
\text { communications. }\end{array}$ \\
\hline 2014 & Yoon et al. [124] & $\begin{array}{l}\text { Video multicast with joint resource allocation and adaptive modulation and coding } \\
\text { in 4G networks. }\end{array}$ \\
\hline 2015 & Wan et al. [125] & $\begin{array}{l}\text { ACM is appealing for underwater acoustic communications to improve the system } \\
\text { efficiency. }\end{array}$ \\
\hline
\end{tabular}

TABLE IV

Milestone of ACM (2010-2015).

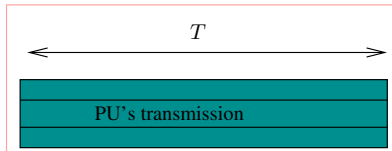

(a)

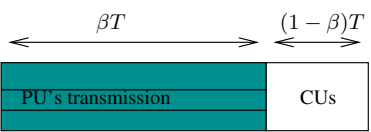

(b)

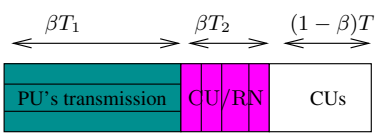

(c)

Time division Channel

(1)

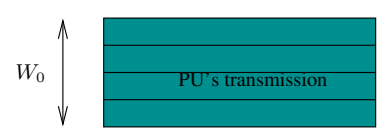

(a)

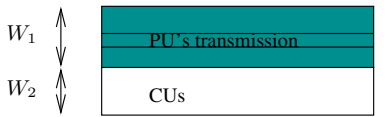

(b)

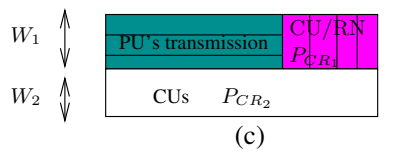

Frequency division Channel

(2)

Fig. 6. The time-division channel versus frequency-division channel in the overlay aided CCR scheme. The bandwidth is $W_{0}=W_{1}+W_{2}$.

in $W_{1}$. In the other frequency band, namely $W_{2}$, the PU/SN remains silent and the other CUs transmit their own signals by using the entire time-slot (TS) T. More specifically, the PU/SN and CU/RN will share the bandwidth $W_{1}$ to convey the source message to the PU/DN, while the other CUs may use the remaining bandwidth of $\left(W_{2}=W_{0}-W_{1}\right)$ for their own communications. The PU/SN transmits using the power of $P_{S}$ during $T_{1}$, while the CU/RN forwards the source message using the power of $P_{C R, 1}$ during $T_{2}$ and the second $\mathrm{CU}$ can broadcast its message to other CUs using the power of $P_{C R, 2}$ during the entire time period $T$, which is illustrated in Fig. 7. During the first TS $T_{1}$, the PU/SN broadcasts the

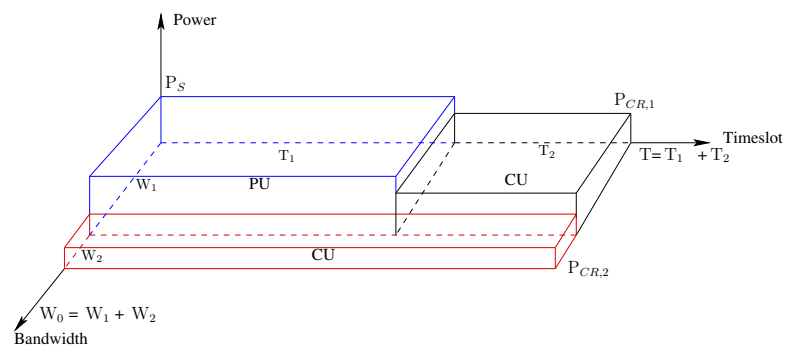

Fig. 7. The bandwidth, time period and power allocation for the PU and CU, which obey the protocols of Fig. 6. The total TS duration is $T=T_{1}+T_{2}$ and the total bandwidth is $W_{0}=W_{1}+W_{2}$ (c) [126].

source message $x$ to both the CU/RN and the PU/DN.

During the second time slot $T_{2}$ the CU/RN would forward the source message to the PU/DN using the transmission power of $P_{C R, 1}$ watts/Hz. Additionally, our CU/RN is capable of carrying out the Decoder-and-forward (DAF) operation. When considering the DAF protocol, provided that the $\mathrm{RN}$ is capable of decoding the transmitted symbol correctly, it forwards the decoded symbol with a power $P_{C R, 1}$ to the DN. Otherwise the RN remains idle. In [127], the PU/SN transmits during $T_{1}$, while the CU/RN transmits during $T_{2}$. Both the PU/SN and CU/RN utilize the bandwidth $W_{1}$. When we consider the DAF protocol, the capacity of our system is limited by the capacity of either the SR link or that of the combined channel constituted by the SD and RD links which ever is lower. Then the Continuous-Input Continuous-Output Memeryless Channel (CCMC) capacity of DAF transmissions over $W_{1} \mathrm{~Hz}$ can be formulated as [pg. 126] [18]:

$$
\begin{gathered}
C_{P U}^{D A F}=\frac{W_{1}}{2} \\
\min \left[\log _{2}\left(1+\frac{P_{S}\left|h_{s d}\right|^{2}}{N_{0}}+\frac{P_{C R, 1}\left|h_{r d}\right|^{2}}{N_{0}}\right), \log _{2}\left(1+\frac{P_{S}\left|h_{s r}\right|^{2}}{N_{0}}\right)\right]
\end{gathered}
$$

The factor $\frac{1}{2}$ in Eq. (3) indicates that the PU only utilizes the first time slot $T_{1}$ of Fig. 7, while the CU uses the second time slot $T_{2}$ to transmit its signals. Without loss of generality, we assume $T_{1}=T_{2}=\frac{T}{2}$.

The bandwidth requirement of $W_{1}$ can be expressed as:

$$
W_{1} \geqslant \frac{2 R_{P U}}{\min \left[\log _{2}\left(1+\frac{P_{S}\left|h_{s d}\right|^{2}}{N_{0}}+\frac{P_{C R, 1}\left|h_{r d}\right|^{2}}{N_{0}}\right), \log _{2}\left(1+\frac{P_{S}\left|h_{s r}\right|^{2}}{N_{0}}\right)\right]}
$$

Therefore, the CU's own data rate using the released bandwidth $W_{2}$ is given by [127]:

$$
R_{C R}=\left(W_{0}-W_{1}\right) \log _{2}\left[1+\frac{P_{C R}\left|h_{C R}\right|^{2}(1-\psi)}{\left(W_{0}-W_{1}\right) N_{0}}\right]
$$


which can be optimized with respect to the power coefficient $\psi$. If the total transmission power of CUs is limited to $P_{C R}$, then we have:

$$
P_{C R}=\frac{1}{2} P_{C R, 1} W_{1}+P_{C R, 2} W_{2} .
$$

The ratio of transmission power allocated for helping the PU/SN to the total transmission power of the CUs over the bandwidth $W_{1}$ is formulated as:

$$
\psi=\frac{\frac{1}{2} P_{C R, 1} W_{1}}{P_{C R}},
$$

In this way, the CUs can decide how to share their joint transmission power in order to maximize their own data rate by using the released bandwidth. The amount of bandwidth savings achieved by the PUs with the aid of the CUs assisted will be discussed in Section V-C.

\section{B. Time-division channel model}

In [128], the time-division channel in CCR scheme has been considered. The time-division channel of Fig. 6 illustrates the time period allocation of the PUs and CUs, where $T$ is the original time period allocated for the $\mathrm{Pt}$ to transmit its source message to the Pr. We will refer to $\beta$ as the time allocation fraction, where $0<\beta<1$. When the $\mathrm{Pt}$ is assisted by a $\mathrm{Ct} / \mathrm{RN}$, the Pt relies on a time-fraction of $\beta T$ to convey the source message to the Pr and $\mathrm{Ct} / \mathrm{RN}$. More specifically, the $\mathrm{Pt}$ simultaneously transmits its message to Pr and $\mathrm{Ct} / \mathrm{RN}$ during the $\beta T_{1}$ time-period. Additionally, the $\mathrm{Ct} / \mathrm{RN}$ cooperatively relays the Pt's signal to $\operatorname{Pr}$ in the subsequent $\beta T_{2}$ time-periods. Then the Pr applies maximum ratio combining for detecting the signal received from the Pt during the first $\beta T_{1}$ time period, and the signal received from the $\mathrm{Ct} / \mathrm{RN}$ in the subsequent $\beta T_{2}$ time periods. After the PU has ceased its transmission, the system will allow the CUs to transmit their information to the other CUs by using the remaining time period of $T_{2}=$ $\left(1-\beta_{l, k}\right) T$ for their own communications. We assume that our proposed scheme relies on a Time Division Multiple Access (TDMA) scheme, where PUs do not transmit simultaneously for the sake of avoiding any inter-user interference. During the second TS $T_{1}$ the $\mathrm{Ct} / \mathrm{RN}$ would forward the source message to the Pr using the transmission power of $P_{C R}$ watts/Hz. The signal received by the Pr via the RD link employing the Amplify and Forward (AAF) protocol. Both the Pt and the $\mathrm{Ct} / \mathrm{RN}$ utilize the same frequency bandwidth. The achievable instantaneous rate of the $l$ th PU when employing the $k$ th $\mathrm{CU}$ at a given $\beta_{l, k}$ may be represented as:

$$
R_{l, k}^{P U}\left(\beta_{l, k}\right)=C_{P U_{l, k}} \beta_{l, k} .
$$

where the capacity of PU $C_{P U_{l, k}}$ based on the Shannan theory is given by:

$$
C_{P U_{l, k}}=\frac{T}{2} \log _{2}\left[1+\frac{\gamma_{P U}\left|h_{P t_{l}, P r_{l}}\right|^{2}}{d_{P t_{l}, P r_{l}}^{\alpha}}+f_{P t, C t, P r}\right],
$$

where we have
$f_{P t, C t, P r}=$
$\frac{\gamma_{P U} \gamma_{C U}\left|h_{P t_{l}, C t_{k}}\right|^{2}\left|h_{C t_{k}, P r_{l}}\right|^{2}}{\gamma_{P U}\left|h_{P t_{l}, C t_{k}}\right|^{2} d_{C t_{l}, P r_{l}}^{\alpha}+\gamma_{C U}\left|h_{C t_{k}, P r_{l}}\right|^{2} d_{P t_{l}, C t_{l}}^{\alpha}+d_{P t_{l}, C t_{l}}^{\alpha} d_{C t_{l}, P r_{l}}^{\alpha}}$.

The factor $\frac{1}{2}$ in Eq. (9) is due to the time fraction $\epsilon_{l, k}=\frac{1}{2}$, when we have $T_{0}=T_{1}$, where the Pt utilizes the first TS $T_{0}$ and the $\mathrm{Ct} / \mathrm{RN}$ uses the second TS $T_{1}$ to transmit the PU's signals. Note that the transmit SNR of the PU is $\gamma_{P U}=\frac{P_{s}}{N_{0}}$ and that of the $\mathrm{CU}$ is $\gamma_{C U}=\frac{P_{C R}}{N_{0}}$.

The achievable transmission rate of the $k$ th $\mathrm{CU}$ when assisting the $l$ th $\mathrm{PU}$ at a given $\beta_{l, k}$ is formulated as:

$$
R_{l, k}^{C U}\left(\beta_{l, k}\right)=\left(1-\beta_{l, k}\right) T \log _{2}\left[1+\gamma_{C U}\left|h_{C t_{k}, C r_{k}}^{(k)}\right|^{2}\right],
$$

where the channel $h_{C t_{k}, C r_{k}}^{(k)}$ depends on the frequency band provided by $C t_{k}$, while the pathloss is $\varrho=1 / d_{a b}^{\alpha}[18]$.

\section{The Bandwidth Reduction of PUs}

The achievable bandwidth reduction in [126] based on four fix-mode transmission schemes are discussed in this section. More specifically, System A in Fig. 8 is a non-cooperative system, while System B, System C and System D are OWR aided CCR systems. We assume that both the SN and the DN are PUs and the RN is a CU. The systematic diagram of OWR aided CCR system which all transmission links experienced the ATTCM has been presented in Fig. 9. As discussed in [126], $\zeta$ of Fig. 8 is the passband bandwidth of PSK/QAM modulation, which is assumed to be the same as the symbol rate of $R_{s} \mathrm{symbol} / \mathrm{s}$. Thus, the bit rate of the system is given by: $R_{b}=\eta \times R_{s}$ (bit/s), where $\eta$ is the throughput in Bit Per Symbol (BPS). The received SNR $\left(\mathrm{SNR}_{r}\right)$ in decibel is given by: $S N R_{r}=S N R_{t}+\tilde{G}$, while the transmit $S N R^{3}$ is expressed as: $S N R_{t}=10 \log _{10}\left(\frac{P_{t}}{N_{0}}\right)$, where $P_{t}$ is the transmit power and $N_{0}$ is the single-sided noise power. We assume that a BER of $10^{-5}$ or less is required at the DN, where received SNRs of $9 \mathrm{dBs}$ and $18 \mathrm{dBs}$ are necessitated at the DN, when TTCM-8PSK and TTCM-64QAM are employed, respectively. The SD link is assumed to be of low quality and hence it is considered to be unavailable in this example. Additionally, System A constitutes our benchmark arrangement for the other three schemes, as seen from Fig. 8. The PU/SN of System $\mathrm{B}$ is capable of increasing its throughput to $\eta_{B}=2.5 \mathrm{BPS}$ from the $\eta_{A}=2$ BPS value of System A, when using the same bandwidth of $\zeta=R_{s}$. Their bit rate is $\frac{R_{b}^{A}}{R_{b}^{B}}=\frac{2 R_{s}^{A}}{2.5 R_{s}^{B}}$ upon assuming that System A and System B have the same symbol rate of $R_{s}^{A}=R_{s}^{B}$, while the relationship of their bit rate is given by: $R_{b}^{B}=\frac{\eta_{B}}{\eta_{A}} R_{b}^{A}=1.25 R_{b}^{A}$. Thus, System $\mathrm{B}$ has a $25 \%$ higher bit rate than System A within the same bandwidth. Then the relationship between the bit rate $R_{b}^{B}$ of System B and the symbol rate $R_{s}^{A}$ of System A is given by: $R_{b}^{B}=1.25 R_{b}^{A}=1.25 \times 2 R_{s}^{A}=2.5 R_{s}^{A}$.

\footnotetext{
${ }^{3}$ The concept of transmit SNR [129] is unconventional, as it relates quantities to each other at two physically different locations, namely the transmit power to the noise power at the receiver, which are at physically different locations.
} 


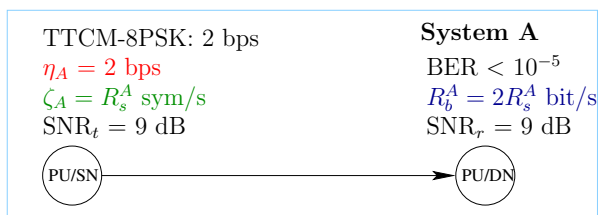

(a)

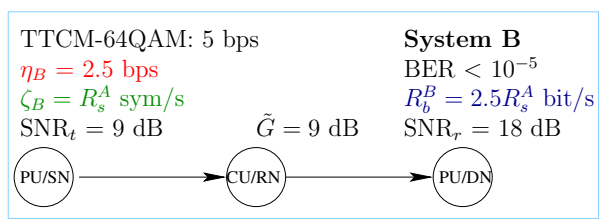

(c)

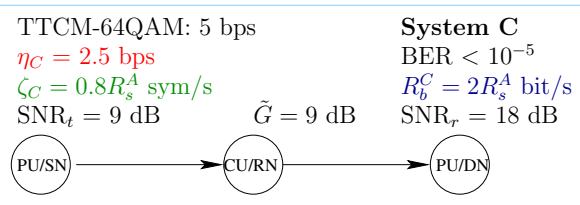

(b)

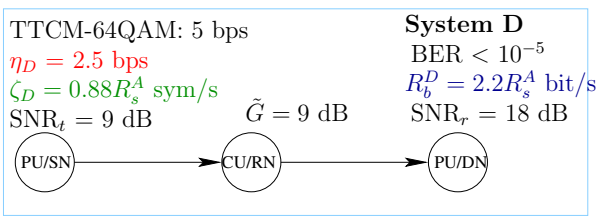

(d)

Fig. 8. Comparison of a non-cooperative scheme and of three relay-assisted DAF-CR schemes, where the target $S N R_{t}$ is $9 \mathrm{~dB}$ since the RN is located at the mid-point between the SN and the DN and the corresponding pathloss exponent is $\alpha=3$. Additionally, the relay-assisted schemes were rely on the protocols of Fig. 7 where the CU helps the PU to transmit its information.

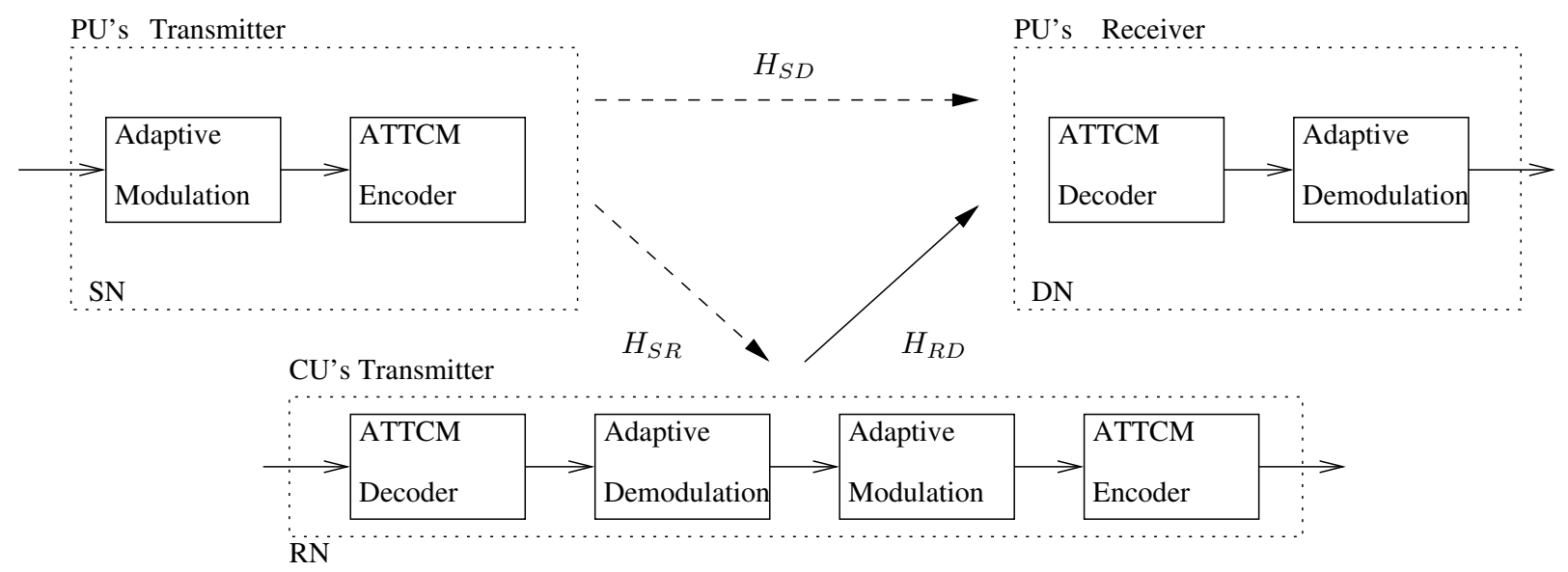

Fig. 9. The architecture of the ATTCM aided OWR in CCR scheme, which obeys Fig. 8, but additionally incorporate by adding the ATTCM components.

By contrast, both System A and System C have the same bit rate of $R_{b}^{A}=R_{b}^{C}$, while the relationship of their symbol rates is given by:

$$
\begin{aligned}
R_{s}^{C} & =\frac{\eta_{A} R_{s}^{A}}{\eta_{C}}, \\
& =0.8 R_{s}^{A}
\end{aligned}
$$

Hence, System $\mathrm{C}$ is capable of providing the same bit rate using only $80 \%$ of the original bandwidth. This is achieved as a benefit of its lower Baud-rate of $\frac{\eta_{A}}{\eta_{C}} R_{s}$, where $\frac{\eta_{A}}{\eta_{C}}=\frac{2}{2.5}=0.8$ is the throughput ratio of System A to System C. Then the relationship between the bit rate of System $\mathrm{C}$ and the symbol rate of System A becomes $R_{b}^{C}=2 R_{S}^{A}$. If we create a System $\mathrm{D}$, where the bit rate of the PU is lower than that of System $\mathrm{B}$, but higher than that of System $\mathrm{A}$, then we have $R_{b}^{D}=$ $1.1 R_{b}^{A}$. By referring to Fig. 8, we have $\eta_{A}=2.0 \mathrm{BPS}$ and $\eta_{D}=2.5$ BPS. Furthermore, we have $R_{b}^{D}=1.1 \eta_{A} \times R_{s}^{A}=$ $1.1 \times 2 \times R_{s}^{A}=2.2 \times R_{s}^{A}$. Based on Eq. (12), we arrive at $R_{s}^{D}=\frac{\eta_{A} R_{s}^{A}}{\eta_{D}}=\frac{2.2}{2.5} R_{s}^{A}=0.88 R_{s}^{A}$. Then the bandwidthreduction factor becomes $B_{s}=1-\frac{\eta_{A}}{\eta_{D}}=1-0.88=0.12$. In this situation, System D is capable of reducing the original bandwidth by $12 \%$ for the CU's benefit, while the PU enjoys an additional 0.5 BPS throughput increment.

The comparisons of these four systems are shown in a nutshell in Table V. As shown in Table V, System B achieves the highest bit rate but uses all available bandwidth, i.e. achieves no bandwidth reduction. By contrast, System $\mathrm{C}$ achieves the highest bandwidth reduction, while maintaining the same bit rate as System A. Furthermore, System D achieved both a practical bit rate improvement as well as some bandwidth reduction.

More specifically, the bandwidth-reduction factor is given by: $B_{s}=1-\frac{\eta_{A}}{\eta_{C}}$. Therefore, we would employ System B as the OWR scheme in our CR, since a CU assisting the PU's transmission is capable of saving the highest amount of $20 \%$ $(1-0.8=0.2=20 \%)$ of the PU's bandwidth among these three relay-assisted OWR schemes.

\section{TWR aided overlay SAS in CCR network}

For the sake of increasing the CU's own data rate by exploiting the bandwidth released by the PUs, as well as increasing the throughput of PUs by using one of these CUs as a RN. In [127], the authors have employed the TWR in the overlay SAS. In a TWR assisted CCR system, where the two PUs act as the SNs and the DNs for each other. The schematic of the MABC-TWR scheme is shown in Fig. 10, which consists of PUs. During the first cooperative transmission period, both PUs transmit their signals simultaneously from their SNs, namely PU/SN 1 and $\mathrm{PU} / \mathrm{SN}_{2}$, to the CU/RN.

As shown in Fig. 10, there are two protocols has been considered, namely Time Division Broadcast Channel (TDBC) 


\begin{tabular}{|l|r|r|r|r|}
\hline Fixed model type & System A & System B & System C & System D \\
\hline Bit-per-symbol $\eta$ & $\eta_{A}=2.0 \mathrm{BPS}$ & $\eta_{B}=2.5 \mathrm{BPS}$ & $\eta_{C}=2.5 \mathrm{BPS}$ & $\eta_{D}=2.5 \mathrm{BPS}$ \\
\hline Bit rate(bit/s) & $R_{b}^{A}$ & $R_{b}^{B}=1.25 R_{b}^{A}$ & $R_{b}^{C}=R_{b}^{A}$ & $R_{b}^{D}=1.1 R_{b}^{A}$ \\
\hline Symbol rate(sym/s) & $R_{s}^{A}$ & $R_{s}^{B}=R_{S}^{A}$ & $R_{s}^{C}=0.8 R_{S}^{A}$ & $R_{s}^{D}=0.88 R_{S}^{A}$ \\
\hline Bit rate / $R_{s}^{A}$ & $\frac{R_{b}^{A}}{R_{s}^{A}}=2.0$ & $\frac{R_{b}^{B}}{R_{s}^{A}}=2.5$ & $\frac{R_{b}^{C}}{R_{s}^{A}}=2.0$ & $\frac{R_{b}^{D}}{R_{s}^{A}}=2.2$ \\
\hline Bandwidth-reduction $\left(B_{s}\right)$ & $1-\frac{\eta_{A}}{\eta_{A}}=0(0 \%)$ & $B_{s}^{B}=B_{s}^{A}(0 \%)$ & $1-\frac{\eta_{A}}{\eta_{C}}=0.20(20 \%)$ & $1-\frac{\eta_{A}}{\eta_{D}}=0.12(12 \%)$ \\
\hline
\end{tabular}

TABLE V

THE PARAMETERS OF FOUR FIXED-MODE CCR SCHEMES.

and Multiple-Access Broadcast Channel (MABC). In the TDBC protocol shown in Fig. 10, there is no interference hence the corresponding complexity at the $\mathrm{RN}$ is kept low. Three time slots are used for two data flows, which are $s_{1} \rightarrow r, s_{2} \rightarrow r$, and $s_{1} \leftarrow r \rightarrow s_{2}$, where $s_{1}$ and $s_{2}$ denote the two primary sources, while $r$ denotes the CU which acts as a RN. By contrast, the MABC protocol requires two time slots for transmitting two data flows, which are $s_{1} \rightarrow r \leftarrow s_{2}$ and $s_{1} \leftarrow r \rightarrow s_{2}$. Since the sources transmit their information simultaneously, the MABC system suffers from self-interference. In our paper, we have invoked an advanced MUD technique at the RN in order to decode both information streams of the SNs and to cancel the selfinterference. Explicitly, in the MABC protocol, two signals were transmitted simultaneously from the two PU/SNs, where each PU has a single antenna. Additionally, we have used the powerful maximum likelihood MUD for detecting the two source signals using a single-antenna aided CU/RN, which constitutes a $(2 \times 1)$-element Multiple-Input Single-Output (MISO) [130] system for the SR links. This powerful MUD was required for eliminating avalanche-like error propagation at the RNs. However, opted for $(1 \times 1)$-element Single-Input Single-Output (SISO) system for the RD link, where each DN employs a single antenna for detecting its wanted signal arriving from the RN. By contrast, in the TDBC protocol, we have a $(1 \times 1)$-element SISO system in the two SR links and a single RD link, because the two SNs use two separate time periods for transmitting their information to the $\mathrm{RN}$, respectively.
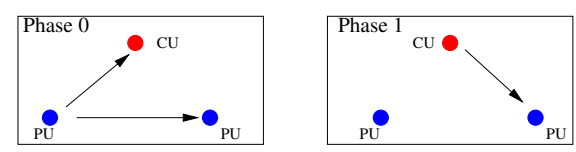

(a) OWR scheme
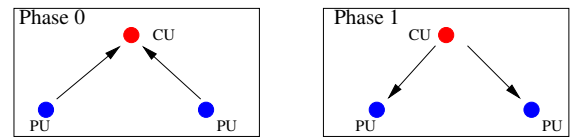

(b) OWR MABC schem
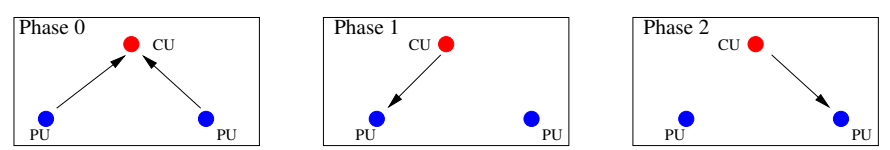

(c) OWR TDBC schem

Fig. 10. The schematic of a TWR-CR system, including two PUs and $K$ CUs. For MABC, it has two transmission phases. For TDBC, it has three transmission phases. The DAF protocols has been employed.

It has opted for appointing the best relay has the set of available $K$ RNs that experience identically and independently distributed (i.i.d) fading. Then the selected best RN decodes and forwards the received signals to the intended destinations, namely to the $\mathrm{PU} / \mathrm{DN}_{1}$ and $\mathrm{PU} / \mathrm{DN}_{2}$, respectively, during the second cooperative transmission period. Hence, the overall system throughput becomes higher than that of a OWR scheme, which requires two TSs for transmitting a single user's information. By employing the TWR protocol, the bandwidth reduction of PUs could be highly improved as shown in Fig. 11. Fig. 11 illustrates how much bandwidth of

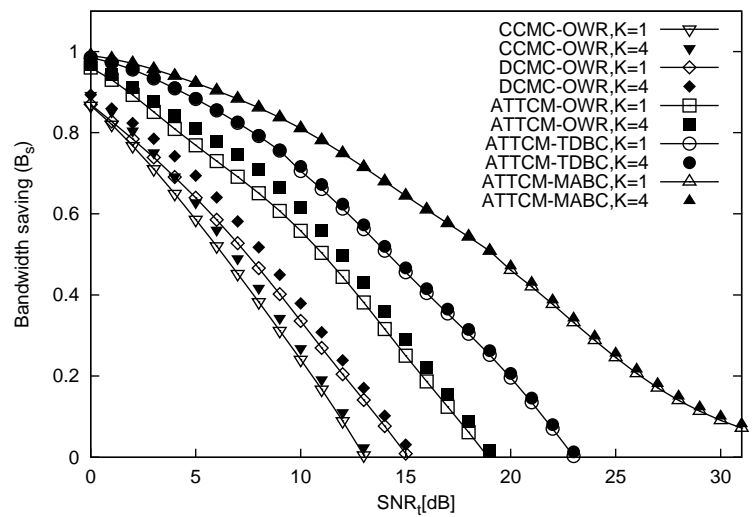

Fig. 11. Bandwidth reduction $B_{s}$ versus $\mathrm{SNR}_{t}$ for the OWR and TWRaided ATTCM schemes in our CCR system communicating over flat Rayleigh fading channels and maintaining a BER below $10^{-5}$. The number of frames simulated was $10^{6} . G_{s r}=G_{r d}=8$, The number of RNs in these schemes is $K=1$ and $K=4$. The idealistic adaptive schemes based on both the Continuous-input Continuous-output Memoryless Channel (CCMC) and on the Discrete-input Continuous-output Memoryless Channel (DCMC) have been considered [131].

the PUs could be saved, when the related relay techniques have been employed in the overlay SAS CCR scheme. In Fig. 11, the attainable bandwidth-reduction $\left(B_{s}\right)$ versus $S N R_{t}$ for the ATTCM, the CCMC and the DCMC aided OWR as well as for the ATTCM-aided TWR schemes have been represented. More specifically, the CCMC based adaptive scheme assumes that idealistic capacity-achieving coding and modulation schemes are employed for communicating exactly at Shannon's capacity. By contrast, the DCMC based adaptive scheme assumes that an idealistic capacity-achieving code is employed for aiding the PSK/QAM modulation schemes considered, for the sake of operating right at the modulation-dependent DCMC capacity. It is interesting to observe that the practical ATTCM scheme is capable of reducing the bandwidth more substantially compared to the idealistic DCMC and CCMC schemes. Furthermore, as the SNR increases, the bandwidth-reduction factor also reduces. This is because when the SNR is high, 
the quality of the SD link is sufficiently high for a fixed transmission throughput of 5 BPS. The inclusion of a RN at high SNRs would only double the transmission period, without actually increasing the transmission throughput. Hence, we are only interested in the operational region, while we have $B_{s}>0$. Fig. 11 also illustrates the attainable bandwidthreduction $\left(B_{s}\right)$ versus $S N R_{t}$ for the ATTCM aided MABC and TDBC TWR system. Since we are only interested in the operational region of $B_{s}>0$, for $S N R_{t}>15 \mathrm{~dB}$, the proposed schemes relying on OWR are no longer beneficial for the range of $B_{s}<0$. The proposed TWR scheme can use the entire bandwidth, since the bandwidth-reduction of the TWR scheme is always higher than zero. Furthermore, the bandwidth reduction can be increased by $88-81=7 \%$ upon employing the TDBC scheme compared to the OWR scheme. Additionally, $92-81=11 \%$ bandwidth reduction can be attained by employing the MABC scheme in comparison to the OWR system. Moreover, at a given SNR, the TWR-CR system always attains a higher $B_{s}$ value, than the corresponding OWR system.

\section{E. Other cooperative techniques for spectrum sharing in CCR network}

Full-duplex transmission constitues a new technology, where the design objective is to allow a node to transmit as well as receive simultaneously, despite the fact that the transmitted signal may have in excess of $100 \mathrm{~dB}$ more power than the received signal. The challenge is to decontaminate the received signal from the leaked transmitted signal, so that an adequate reception can be achieved. Recent research and development on full-duplex relaying has attracted increasing attention in CCR networks for the sake of exploiting that fullduplex relaying offers a high spectral efficiency compared to half-duplex relaying - again, by transmitting and receiving signals simultaneously using the same channel [132]-[134]. In [132] the problem of beamforming optimization in a fullduplex cognitive cooperative energy harvesting network has been considered, in which the $\mathrm{Ct}$ harvests energy from the Pt and relays the information for the PU with the aid of the AAF relaying protocol. Based on the radically new concept of recycling the energy of the self-interference for harvesting energy for the relay, the authors developed a semidefinite programming relaxation method for solving the associated beamforming problem that achieves a significant rate gain over the more conventional power-splitting relaying protocol, which plits the received power between the informationreceiver and the energy-harvesting buffer constituted by the rechargeable battery. The main objective of [133] is to consider full-duplex spectrum sharing assisted cooperative systems relaying on a limited transmit power, when communicating over frequency selective fading environments. The frequency selective fading was counteracted by OFDM transmissions. As a further development, a novel adaptive transmission scheme was designed for cognitive DAF relaying networks in [134]. More explicitly, before each transmission one out of three transmission modes was dynamically selected for maximizing the near-instantaneous capacity of the system, which were half-duplex, full-duplex and no cooperation. Again, energy harvesting constitutes a promising technique of significantly prolonging the battery-recharge of wireless terminals. Cooperative spectrum sharing designed for $\mathrm{CR}$ networks relying on wireless energy harvesting has been studied for example in [135], where the CUs exploit both their energy transfer and relaying capability for improving the performance of the primary system in exchange for gaining access to some of the unused spectrum.

\section{GAME MOdel FOR OVERlay SAS IN CCR NETWORK}

In CR networks, researchers tend to use three major utility functions: i) maximizing the utility of the PUs [136], [137], ii) maximizing the utility of the CUs [138]-[141], and iii) maximizing the total utility of both PUs and CUs [142][145]. In this section, we discuss a fairly general cooperative spectrum sharing technique conceived for maximizing the PUs' utility, and then provide a solution in the form of a distributed algorithm that can be shown to be convergent but sub-optimal.

\section{A. Non-cooperative game}

In the non-cooperative game model, each user only cares about its own benefits and chooses the optimal strategy for maximizing its own payoff. The users are acting selfishly, thus the strategy of each user is developed and planned from the users' own perspectives, which is far from optimum in terms of a holistic network-oriented perspective. Additionally, there is no centralized authority. A novel spectrum access mode was proposed in [146], which enables distributed and opportunistic access to spectral resources using a non-cooperative game theoretic approach, which strikes an appealing tradeoff between the time of access to the channel and the interference imposed on the PU. To elaborate a little further, a pair CUs who want to access the spectrum act in a non-cooperative manner, which implies that they may compete with each other for the limited spectral resources.

To elaborate a little further, in [147], an auction-based mechanism has been proposed for an overlay SAS CR scheme, where the CUs represent the bidders, who are in the position to decide the transmit power that they are willing to employ for relaying the PU's data. Additionally, the PUs have the role of the auctioneer deciding both the total duration of the spectrum lease as well as the leasing time allocated to each CU. More specifically, each CU places a bid representing the specific transmit power's fraction that it is willing to devote for relaying the PU's message. Therefore, the CUs participate in a non-cooperative power control algorithm, where each $\mathrm{CU}$ chooses its specific transmit power fraction in a bid to maximize its own utility. By contrast, each Pt acts as an auctioneer, who decides upon the leasing time $T_{l}$ as well as upon the time $T_{a}$ allocated to each $\mathrm{CU}$, according to the collected bids, namely $T_{l}=\sum_{k=1}^{K} T_{a}(k)$. The spectrum leasing time of each $\mathrm{CU}$ is assumed to be proportional to its contribution to the cooperative process of relaying the PUs' message. Therefore, the leasing process aims for optimizing the performance of both the PUs and CUs. Furthermore, an auction-based power-allocation scheme is proposed for solving 
the 'power competition' of multiple CUs in [148], where multiple CUs transmit via a common relay and compete for the transmit power of the relay in the overlay SAS scheme. Specifically, the Cr cannot always successfully decode the Ct's signal. If the signal of the $\mathrm{Ct}$ is not decided correctly by the $\mathrm{Cr}$, the $\mathrm{Cr}$ is unable to help, hence the $\mathrm{Ct}$ would not join the current auction, but it can attempt the same action again in the next frame. By contrast, the $\mathrm{Ct}$ whose data was successfully recovered at the $\mathrm{Cr}$ is allowed to participate in the current auction. During the auction game, the bid update and power allocation processes are iterated in an alternating fashion, until the auction game converges to the optimum.

Therefore, the Stackelbeg game [104] can be invoked for modelling the behavior of independent decision makers, which constitutes a popular distributed approach. To elaborate a little further, the Stackelberg model constitues a "leader-follower" oriented strategic game in which there is at least one leadplayer, who can make a decision and commit to the specific strategy based on the price. The players engage in Stackelberg competition, provided that at least one of them has an incentive to make a move first. The followers can then adopt the strategy leading to maximizing the profit.

To elaborate a little further, a Stackelberg game based cooperative waiting-time reduction method was proposed for an overlay SAS scheme in [149]. The users seeking admission to a network engage in cooperation and trading with the PUs, with the objective of purchasing unused time for unlicenced access. Additionally, the optimal relay power and the timeduration fractions can also be determined with the aid of the Stackelbeg game. In the proposed algorithm the $(\mathrm{Pt}, \mathrm{Ct})$ pair of a given transmission frame acts as a 'one-leader one-follower' regime. Specifically, a PU may act as a leader who wants to obtain the highest possible benefit from trading, while the CU may decide to cooperate for reducing its transmission time and energy. As a further development, the authors of [150] consider a property-rights models where a PU can lease its own spectrum to a certain number of CUs in exchange for cooperation. More specifically, the PUs lease their spectrum for the sake of maximizing their own quality of service by relying on the CU's assistance, while the CUs first decide, whether to cooperate and if so, they compete among themselves for a transmission opportunity by obeying a distributed power control mechanism.

A similar study was disseminated in [151], where the PU sets the spectrum price for the sake of maximizing its own utility, whereas the CUs choose the power levels to be used for their cooperation with the PU so as to determine the corresponding spectrum access time. According to the sequential structure of decision making, the authors of [152] analyzed the established CCR using a two-stage Stackelberg game under the assumption of having perfect side-information and demonstrated that the utility of both the PU and of the CUs becomes higher than that of the corresponding non-cooperative benchmarker. Furthermore, a potential game conceived for overlay-based SAS schemes has been discussed in [70], where the decisions are carried out by the CUs in a distributed manner with the objective of maximizing their individual utility and these decisions depend on those made by the other
CUs. It was demosntrated that the proposed game is always capable of reaching a pure Nash equilibrium. Finally, a specific game has been conceived for overlay-based SAS scheme aiming for regulating the channel occupancy of CUs [153]. The main objective is to maximize the utility of each individual $\mathrm{CU}$, which is equal to the difference between the average throughput of a CU required for transmitting at a specific rate and the associated cost that is proportional to the fraction of time during which a user occupies the channel.

\section{B. Matching game model}

Matching theory [154], [155] has been widely used as an efficient technique of solving the combinatorial problem of matching players of a pair of distinct sets by exploiting the players' individual information and their preferences [156]. A widely-used technique of studying the interactions between a pair of disjoint player sets relies on a so-called twosided market setting. In matching theory, both the cooperative interactions between the users in these distinct sets as well as the competitive interactions amongst the users of the same set are considered [157]. The classical matching problems may be subdivided into one-to-one matching [136], many-toone matching and many-to-many matching regimes [158]. By definition, in the one-to-one matching scenario each player can only be matched to a single member of the opposite set. Naturally, at least one player of the many-to-one matching problem can be matched to multiple players in the opposite set, whilst in the context of many-to-many matching, the players in each of the two sets can be matched to more than one member from the other set. The distributed algorithms of [61], [136] belong to the one-to-one matching problems of overlaybased SAS CCR. The definition of the associated preference list will be introduced in Section VI-B.1 before discussing the corresponding matching game.

1) Preference List: Before any offer is made to the CUs, the PUs construct a preferred list of CUs, which are capable of satisfying the PU's rate requirement. Specifically, each Pt has a preference list of $\mathrm{Cts} / \mathrm{RN}$ that may assist in relaying its message, so that its achievable sum rate becomes higher than its minimum sum-rate requirement. Thus, the preference list for $\mathrm{Pt}_{l}$ is given by:

$$
P U L I S T_{l}=\left\{\left(C t_{\kappa(k)}, C r_{\kappa(k)}\right)\right\}_{k=1}^{L_{C U}},
$$

where the function $\kappa(k)$ satisfies the following conditions:

$$
R_{l, \kappa(k)}^{P U}\left(\beta_{l, \kappa(k)}\right)>R_{l, r e q}^{P U}, k \in\left(1, \ldots, L_{C U}\right) .
$$

The index of the CUs may be recorded in the PULIST, while their corresponding rate has satisfied the PU's rate requirement. Additionally, we have assumed that the first $\mathrm{Ct}_{\kappa}(k)$ at the top of the PULIST $T_{l}$ provides the highest rate $R_{l, \kappa(k)}^{P U}\left(\beta_{l, \kappa(k)}\right)$. Similarly, each CU also has its preferred PU list, and if it transmits in the spectral band occupied by the preferred PUs then its achievable transmission rate is higher than its minimum sum-rate requirement, $R_{k, r e q}^{C U}$. Thus, the preference list for $\mathrm{Ct}_{k}$ is given by:

$$
C U L I S T_{k}=\left\{\left(P t_{\iota(l)}, \operatorname{Pr}_{\iota(l)}\right)\right\}_{l=1}^{L_{P U}}
$$


where the function $\iota(l)$ satisfies the following conditions:

$$
R_{\iota(l), k}^{C U}\left(\beta_{\iota(l), k}\right)>R_{k, r e q}^{C U}, l \in\left(1, \ldots, L_{P U}\right) .
$$

Again, the ordering of the CULIST $_{k}$ also ranges from the highest to the lowest.

2) Matching Algorithm: In [136], a Conventional Distributed Algorithm (CDA) was proposed, which may be viewed as an evolution from the dynamic auction algorithms [159], [160], since it considers a resourceallocation [161] framework that facilitates a joint competitive strategy of the PUs and the CUs conceived for accessing the spectral resources. Moreover, the authors of [136] conceived a non-cooperative game, which employs the CDA for efficiently representing the interaction among the competing PUs, where each PU chooses its allocation independently of the others in order to improve its own performance. This is considered as our first scenario, where the PUs do not cooperate with each other. Explicitly, a spectral access strategy is designed for multiple PUs and CUs, where the PUs and CUs are carefully paired for ensuring that both the PUs' and the CUs' minimum sum-rate requirements are satisfied. Each paired $\mathrm{CU}$ assists in relaying its paired-PU's signal in exchange for a transmission opportunity using the PU's spectrum. However, the PUs under the CDA would sometimes compete among themselves for cooperating with the same relay, which may degrade both their utility and throughput. The key idea of CDA algorithm is that each (Pt, Pr) pair trades with a particular $(\mathrm{Ct}, \mathrm{Cr})$ pair for the sake of attaining mutual benefits in the context of cooperative relaying. More specifically, the CDA constitutes a non-cooperative scheme, where none of the PUs cooperates. Instead, they compete with each other, with the selfish objective of maximizing their own rate. In order to explicitly portray the process of this algorithm, we conceived a simple example, which has two PUs and two CUs, as shown in Fig. 12. Each PU has its preference list and its minimum

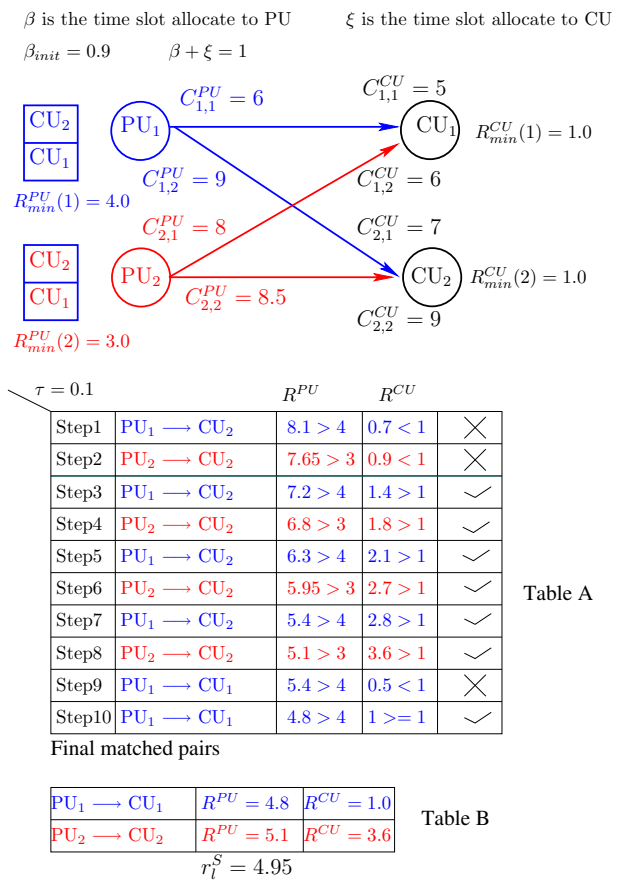

Fig. 12. A example to illustrate the CDA algorithm. rate requirement $R_{\text {min }}^{P U}(l), l \in L$. Note that the PU's achievable sum rate would not be satisfied, if it is lower than the minimum rate requirement. Each PU makes an offer to its most favored $\mathrm{CU}$. If a CU receives two offers, it would select the one that may provide a higher TS for itself. The TS allocated to the $\mathrm{CU}$ of Fig. 12 is denoted by $\xi$, where we have $\xi=1-\beta$. In our example, $\mathrm{CU}_{2}$ is the top candidate in the PULISTs of $\mathrm{PU}_{1}$ and $\mathrm{PU}_{2}$. As shown in Table $\mathrm{A}$ of Fig. 12, during Step1 and Step2 both $\mathrm{PU}_{1}$ and $\mathrm{PU}_{2}$ make their offers to $\mathrm{CU}_{2}$ with the initial TS allocation of $\beta_{\text {init }}=0.9$. Additionally, $\mathrm{CU}_{2}$ chose $\mathrm{PU}_{2}$ at Step8 by rejecting its initial match to $\mathrm{PU}_{1}$ during Step7. This is because choosing $\mathrm{PU}_{2}$ may provide $R_{2,2}^{C U}=C_{2.2}^{C U} \xi=9 \times(1-0.6)=3.6$ (which is higher than $R_{2,1}^{C U}=C_{2,}^{C U} \xi=7 \times(1-0.6)=2.8$ gleaned from $\left.\mathrm{PU}_{1}\right)$ for $\mathrm{CU}_{2}$. Specifically, the value of $\beta$ would be reduced to 0.6 (where $\left.\beta_{\text {init }}-3 \tau=0.9-(3 \times 0.1)=0.6\right)$ if the first offer in the previous step is not accepted. In the CDA, $\mathrm{PU}_{1}$ and $\mathrm{PU}_{2}$ will compete with each other by increasing the TS allocation of $\xi$ and by reducing the value of $\beta$, until one of them loses out, namely when its TS budget would result in $R_{l, k}^{P U}<R_{m i n}^{P U}$. Hence, if two PUs are in favor of the same $\mathrm{CU}$, the competition would fail to bring about any benefits for the competing PUs. The final matched pairs are shown in Table B of Fig. 12.

\section{Cooperative game}

In cooperative games, a centralized controller is invoked for managing the game globally and the users within a group are assumed to be cooperative rather than competitive, with the goal of maximizing the group's total utility. Cooperative game theoretic investigation of spectrum access and sharing problems were investigated based on either Nash Bargaining solutions [108]-[111] or on coalition games [106], [107]. The Nash bargain solution is used for formulating the interaction amongst the cooperative players - provided that a player is capable of influencing the actions of other player, whil;st simultaneously ensuring both efficiency as well as fairness amongst the players. In [162], the CUs are oragnized in groups and form coalitions with the objective of improving the total spectral efficiency of the coalition in overlay-based SAS schemes. Additionally, novel rules are introduced for forming different coalitions amongst the coexisting PUs, where the final coalition structure is shown to be stable. Provided that all the CUs were reassigned to the same coalition, all of them would transmit over the same channel along with the coexisting PUs. To elaborate a little further, the objective is to form different coalitions for the CUs around each PU in the network, so that the CUs of each coalition succeed in improving their spectral efficiency while reducing the interference imposed on each other and on the coexisting PU. Therefore, the CUs have no interest in forming a grand coalition. Additionally, a coalition formation game has been discussed in the context of an overlay-base SAS-CCR scheme in [163] in order to improve the overall system performance and also to encourage cooperation amongst the CUs, which generates interference among themselves. Therefore, coalition game theoretical approaches allow CUs to form coalitions, which cooperatively mitigates the interference and improves the attainable spectrum efficiency. Moreover, a cooperative game model called PDA has been proposed in [61], which was designed for supporting the efficient spectral access of multiple PUs and CUs in CCR networks. This PDA may be classified as a combination game model of repeated game [98], [164], [165] and coalition game, where all PUs are capable 
of cooperating with each other, this constitutes the second scenario as described in Section VI, where the PUs do not compete with each other. The PUs are motivated to form a grand coalition [166], [167] for achieving an increased expected PU rate by discouraging the PUs from competing with each other for the same CU's assistance. Furthermore, the concept of a penalty/punishment is introduced [164], which is imposed only for a carefully selected finite period for the sake of discouraging non-cooperation among the PUs. Moreover, in [61], it was shown that this PDA reaches an equilibrium, when it is repeated for a sufficiently long duration. These benefits are achieved, because the PUs are motivated to cooperate by the incentive of achieving a higher PU rate, whilst again, non-cooperation can be discouraged with the aid of a limited-duration punishment. More specifically, a game unit is constituted by $L_{P U}$ rounds and each round has $L_{P U}$ transmissions, where the PUs take turns to select the best available CU according to a round-robin type priority access list. The priority access list of the $i$ th round is given by:

$$
A L I S T_{i}=\left\{P t_{i}, P t_{i \oplus 1}, \ldots, P t_{l}, \ldots, P t_{i \oplus\left(L_{P U}-1\right)}\right\}
$$

where $i=\left\{1,2, \ldots, L_{P U}\right\}$ and the subscript of $P t_{l}$ for the $j$ th transmission $\left(j=\left\{1,2, \ldots, L_{P U}\right\}\right)$ in the $i$ th round is based on the modulo- $L_{P U}$ summation:

$$
l=i \oplus(j-1)=(i+(j-1)) \bmod L_{P U} .
$$

Hence, we have $A L I S T_{1}=\left\{P t_{1}, P t_{2}, P t_{3} \ldots, P t_{L_{P U}}\right\}$ and $A L I S T_{2}=\left\{P t_{L_{P U}}, P t_{1}, P t_{2}, \ldots, P t_{L_{P U-1}}\right\}$. The first Pt in the $A L I S T_{i}$ has the first priority to select its best CU. Then the second $\mathrm{Pt}$ in the list selects the best available $\mathrm{CU}$ from the remaining set of CUs, while the third Pt in the list selects its best available $\mathrm{CU}$ afterwards and the same procedure is invoked for the rest of the Pts in the list. During the next round, the first $\mathrm{Pt}$ in $A L I S T_{i}$ will become the second $\mathrm{Pt}$ in $A L I S T_{i+1}$, while the last $\mathrm{Pt}$ in $A L I S T_{i}$ is now the first $\mathrm{Pt}$ in $A L I S T_{i+1}$ according to the round-robin scheduling. Hence, after $L_{P U}$ rounds each PU is guaranteed to have access to $\min \left\{L_{P U}, L_{C U}\right\}$ CUs amongst the top CUs in its PULIST ${ }^{4}$, but is has no access to any CUs for the remaining ( $L_{P U}-$ $L_{C U}$ ) transmissions. In this way, the PUs give up any futile competition and cooperatively take turns, one at a time, to access the available CUs, which is expected to yield the most benefits for themselves. If none of the CUs in the current list may be satisfied, then only this specific Pt will update its TS allocation and then produces a new preference list. The specific details of the algorithm can be summarized as follows: Let us now consider the specific example as shown in Fig. 13, which has the same parameters as those of Fig. 12, where we have two PUs and two CUs. Our game unit has $L_{P U}=2$ rounds and each round has $L_{P U}=2$ transmissions. The first round of our game is shown in Table A of Fig. 13. At Step 1 of Round $1, \mathrm{PU}_{1}$ has a higher priority to select its best candidate $\mathrm{CU}_{2}$, which allows $\mathrm{PU}_{1}$ to update its TS allocation, until they become matched at Step 2 of Round 1 of Fig. 13. After that, $\mathrm{PU}_{2}$ selects its best candidate from the CUs which are not matched, thus the unmatched $\mathrm{CU}_{1}$ has been chosen and the Round 1 match was successfully carried out at step 4. Now, at Round 2, $\mathrm{PU}_{2}$ acquired a higher priority to select its top candidate $\mathrm{CU}_{2}$ at Step 1 of Table B in Fig. 13. Meanwhile, $\mathrm{PU}_{1}$ selects the remaining $\mathrm{CU}_{1}$ at Step 3 of Table B.

\footnotetext{
${ }^{4}$ Provided that the rate requirements in Eq. (14) and Eq. (16) are satisfied.
}

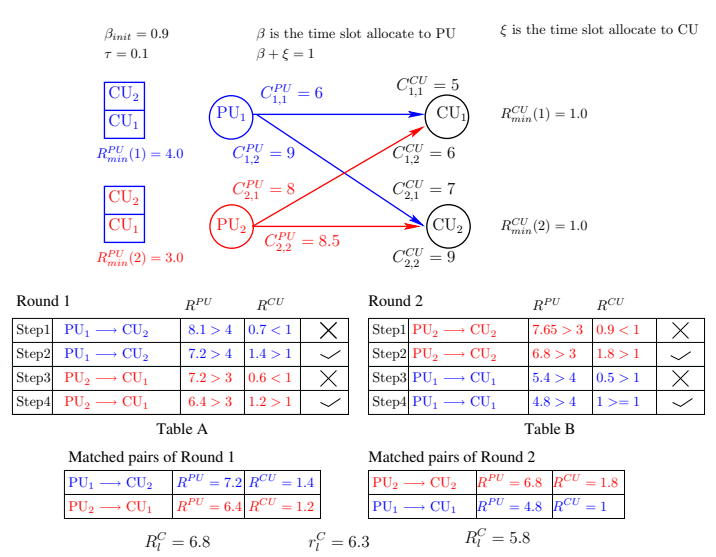

Fig. 13. A example to illustrate our proposed PDA algorithm.

However, we have compared this PDA and one of the existing non-cooperative games, which has been presented in [136]. Additionally, we found that when relying on the same parameters and same system model, the proposed technique has a better performance. Furthermore, we used two additional benchmarkers. The upper-bound of Fig. 14 is constituted by the centralized solution relying on the exhaustive search. Observe furthermore that the distributed solution tend to closely approximate the centralized philosophy. Furthermore, the lower-bound benchmark of Fig. 14 represents the random case.

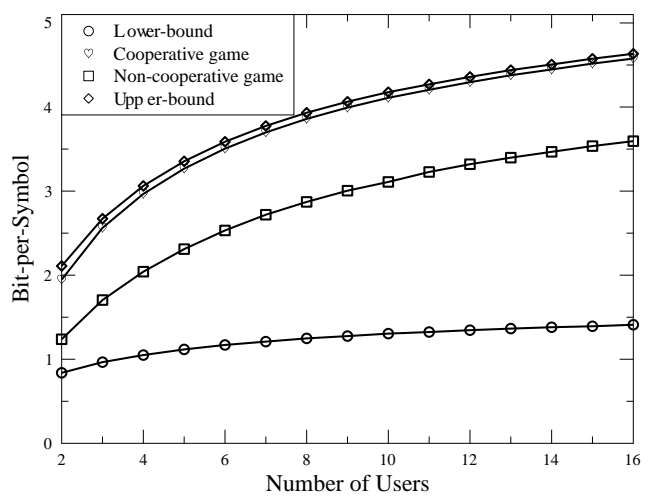

Fig. 14. Comparison of PDA and a existing non-cooperative game of [136].

\section{Comparison of Non-cooperative and cooperative game}

Based on the state-of-the-art in both non-cooperative and cooperative game models described in Section VI-A and Section VI-C, it transpires that the benefit of cooperative games is that the players cooperatively come to an agreement based on bargaining with each other in order to maximize the benefits gleaned. This cooperative gain is typically higher than playing without cooperation. We have discussed a simple example for comparing these two models. In order to investigate more for our proposed cooperative $\mathrm{CR}$ scheme, we have further considered four modes of different coordinates among the $P t$, $\mathrm{Pr}, \mathrm{Ct}$ and $\mathrm{Cr}$ nodes as shown in Fig. 15, which are the fixed pathloss setting. Then the parameters of coordinates of $\mathrm{Pt}, \mathrm{Pr}, \mathrm{Ct}$ and $\mathrm{Cr}$ nodes are shown in Table. VI. Specifically, 

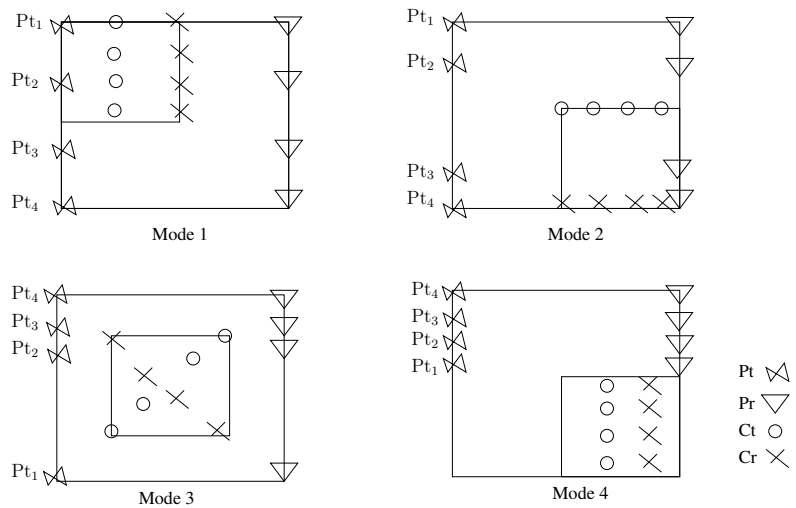

Fig. 15. The schematic of four fixed pathloss distributions based on Table VI.

\begin{tabular}{|c|r|r|r|r|}
\hline Mode ID & Coordinates & $y_{1}=y_{2}$ & $C t:\left(x_{3}, y_{3}\right)$ & $C r:\left(x_{4}, y_{4}\right)$ \\
\hline \multirow{4}{*}{ Mode 1 } & $\mathrm{PU}_{1}$ & 2.0 & $(0.5,2.0)$ & $(1.0,2.0)$ \\
\cline { 2 - 5 } & $\mathrm{PU}_{2}$ & 1.2 & $(0.5,1.6)$ & $(1.0,1.6)$ \\
\cline { 2 - 5 } & $\mathrm{PU}_{3}$ & 0.8 & $(0.5,1.2)$ & $(1.0,1.2)$ \\
\cline { 2 - 5 } & $\mathrm{PU}_{4}$ & 0.0 & $(0.5,1.0)$ & $(1.0,1.0)$ \\
\hline \multirow{4}{*}{ Mode 2 } & $\mathrm{PU}_{1}$ & 2.0 & $(1.0,1.0)$ & $(1.0,0.2)$ \\
\cline { 2 - 5 } & $\mathrm{PU}_{2}$ & 1.5 & $(1.25,1.0)$ & $(1.25,0.2)$ \\
\cline { 2 - 5 } & $\mathrm{PU}_{3}$ & 0.4 & $(1.75,1.0)$ & $(1.75,0.2)$ \\
\cline { 2 - 5 } & $\mathrm{PU}_{4}$ & 0.0 & $(2.0,1.0)$ & $(2.0,0.2)$ \\
\hline \multirow{4}{*}{ Mode 3 } & $\mathrm{PU}_{1}$ & 0.0 & $(1.5,1.0)$ & $(2.0,1.0)$ \\
\cline { 2 - 5 } & $\mathrm{PU}_{2}$ & 1.0 & $(1.5,0.8)$ & $(2.0,0.8)$ \\
\cline { 2 - 5 } & $\mathrm{PU}_{3}$ & 1.5 & $(1.5,0.6)$ & $(2.0,0.6)$ \\
\cline { 2 - 5 } & $\mathrm{PU}_{4}$ & 2.0 & $(1.5,0.4)$ & $(2.0,0.4)$ \\
\cline { 2 - 5 } & $\mathrm{PU}_{1}$ & 1.0 & $(0.8,1.8)$ & $(1.8,0.8)$ \\
\cline { 2 - 5 } & $\mathrm{PU}_{2}$ & 1.25 & $(1.2,1.5)$ & $(1.2,1.0)$ \\
\cline { 2 - 5 } & $\mathrm{PU}_{3}$ & 1.75 & $(1.6,1.0)$ & $(1.6,1.5)$ \\
\cline { 2 - 5 } & $\mathrm{PU}_{4}$ & 2.0 & $(1.8,1.0)$ & $(1.8,1.5)$ \\
\hline
\end{tabular}

TABLE VI

THE PARAMETERS OF SETTING $d_{P t_{l}, C t_{k}}=\sqrt{\left|x_{3}\right|^{2}+\left|y_{4}-y_{1}\right|^{2}}$ FOR FOUR FIXED MODES AS SHOWN IN FIG. 15.

in this design, we also assumed that Pt and Pr are located at the opposite sides of a square at a normalized distance of two, which leads to $x_{1}=0$ and $x_{2}=2$. The distance between the $\mathrm{Cts}$ and $\mathrm{Crs}$ is given by:

$$
d_{C t_{k}, C r_{k}}=\sqrt{\left|x_{4}-x_{3}\right|^{2}+\left|y_{4}-y_{3}\right|^{2}} .
$$

Moreover, the corresponding rate of matched PU with fixed pathloss are shown in Fig. 16 and Fig. 17, when consider the PDA, the CDA algorithms. In Fig. 16, we consider two CUs, which is lower than the number of PUs, namely $K>L$. At each mode, we presented the individual average rate of each PU. The "Average" of Fig. 16 denotes the average rate of total PUs at each mode.

Additionally, "Average sum rate of matched PUs" is the value of $R_{\text {match }}^{P U}$. Observing in Fig. 16(a) and Fig. 16(b), there are up to 2 modes that the average sum rate of matched PUs are higher than the average rate among PUs, which is $R_{\text {match }}^{P U}>\overline{R_{l, k}^{P U}}$, since the number of CUs is two. In Fig. 17(a) and Fig. 17(b), the satisfied modes were increased to 3 , since the number of CUs is increased by comparing to Fig. VI. Whereas, the performance of our proposed PDA is also better than that of the CDA algorithm. By observing the Fig. 17(a), the average sum rate of matched PUs is higher than their individual rate at mode 2 and 4 by employing the PDA algorithm. Note that, the benefit of attending the cooperation among PUs is that they could gain more finally. The CA achieves the highest average total sum-rate among these four algorithms, while the RA achieves the lowest sum rate in Fig. 16 and Fig. 17. It is observed in Fig. 17 that the PDA achieves a higher sum-rate than that of the CDA when the number of PUs and CUs is identical. The PDA consistently attains a higher rate than the CDA for the scenario, where the number of CUs is higher than that of the PUs. As shown in Fig. 16, it is observed that the rate of $\mathrm{PU}_{l}$ operating under our PDA is lower than that of the CA, but much higher than that of the CDA, when the number of PUs is higher than that of the CUs. Furthermore, when the number of $\mathrm{CU}$ is lower than that of the PUs, the CDA scheme performs slightly worse, which is a consequence of the competition loss encountered. Additionally, the average individual PU rate was found to be exactly a fraction of $\frac{1}{L_{P U}}$ of the total PU sum rate for all PUs. Thus the trend of the individual PU rate follows the trend of the total PU sum rate. Hence, the proposed PDA outperforms the CDA in terms of both the total PU sum rate and the individual PU rate, especially when the number of PUs is higher than that of the CUs.

\section{CONCLUSIONS AND DESIGN GUIDELINES}

In this paper, we have reviewed various overlay SAS based CCR schemes. In the overlay SAS, the CUs use part of their power for helping the PUs transmit their information. In return, the PUs would lease some fraction of their frequency bands or time-slots to CUs for their own transmissions. Our design guidelines can be summarized as follows:

- We assumed that there are two schemes in SAS which was observed in Fig. 6, namely the frequency division channel and time division channel. In Section V, we have reviewed the family of cooperative relay techniques in the frequency division channel assisted SAS based on CCR system. For the sake of reducing the transmission bandwidth of PUs and lease more of the PU's bandwidth to the CUs for their secondary transmission, the MABC and TDBC based TWR system has been investigated in Section V-D.

- Moreover, the PUs negotiate with CUs concerning the TS allocation by using game-theoretic techniques as discussed in Section VI. Therefore, the non-cooperative game-theoretic model of Section VI-A as well as the cooperative game-theoretic model of Section VI-C have beed discussed, where the PUs make their decision independently in the context of the non-cooperative model. By contrast, the PUs cooperative with each other in the cooperative model.

Additionally, there are four fixed transmission modes of Fig. 8 have been compared in Section $\mathrm{V}-\mathrm{C}$ for the sake of finding a suitable OWR aided CCR scheme that was capable of saving the highest bandwidth. Thus, System C is of Fig. 8 the most suitable system that is capable of reducing the PUs' bandwidth by $20 \%$ for the CU's benefit. Moreover, in order to achieve a higher bandwidth reduction by the PUs, the TWR technique has been widely employed. Specifically, in 


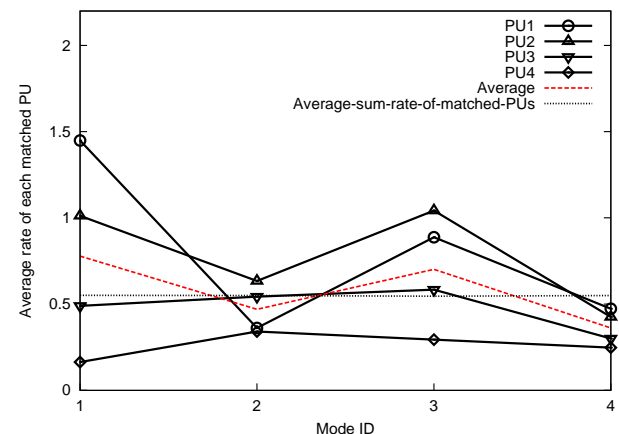

(a) Cooperative game model: PDA

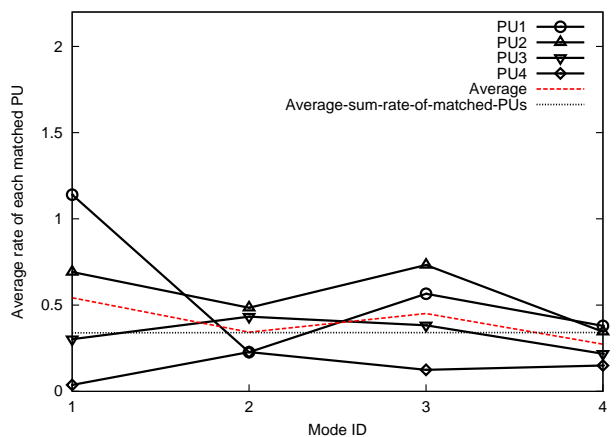

(b) Non-cooperative model: CDA

Fig. 16. Performance of the CCMC aided AAF based cooperative CR scheme communicating over quasi-static Rayleigh fading channel by considering 4 fixed pathloss distribution of Fig. 15 while $L_{P U}=4$ and $L_{C U}=2, \gamma_{C U}=15 \mathrm{~dB}$.

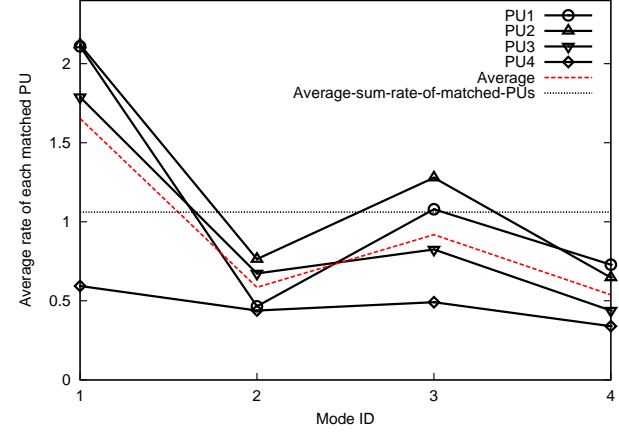

(a) Cooperative model: PDA

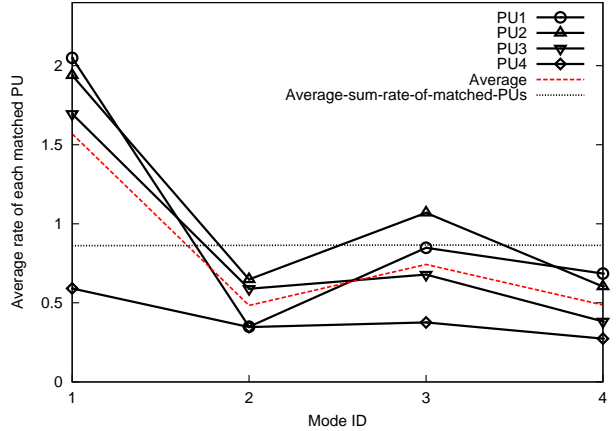

(b) Non-cooperative model: CDA

Fig. 17. Performance of the CCMC aided AAF based cooperative CR scheme communicating over quasi-static Rayleigh fading channel by considering 4 fixed pathloss distribution of Fig. 15 while $L_{P U}=4$ and $L_{C U}=4, \gamma_{C U}=15 \mathrm{~dB}$.

Fig. 11 the bandwidth reduction was increased by $7 \%$ upon employing the TDBC based scheme compared to the OWR scheme. Additionally, an $11 \%$ bandwidth reduction can be attained by employing the MABC based scheme in comparison to the OWR system. Some cooperative techniques conceived for overlay spectrum access scheme has been discussed in Section V-E.

Game theory has been widely employed in overlay SAS aided CCR systems. In Section VI, three types of game models have been surveyed, namely non-cooperative games, matching games and cooperative games. The user in the non-cooperative game model of Section VI-A aims for maximizing its own utility. Several complenning game models have been disseminated in the literature, such as the auction game and Stackelberg's game. Moreover, the benefits of matching theory in overlay SAS schemes have been discussed in Section VI-B.2. To elaborate a little further, the PUs would negotiate with the CUs for the sake of achieving a win-win scenario. Furthermore, the CDA matching algorithm aiming for maximizing the benefits of both the PU and CU has also been discussed. In Section VI$\mathrm{D}$ the PDA cooperative game has been compared to traditional non-cooperative games, where the CUs of the PDA would like to bargain with the PUs for the sake of gaining access to a partial TS with the goal of transmitting their own information. The priority of PUs was carefully protected, so that the PUs' sum rate will be increased with the aid of the CUs. Explicitly, spectrum sharing between the PUs and CUs may be sustained for a long period of time in PDA, which may be viewed as a game repeated for numerous rounds, in which the PUs cooperate based on their individual reputation and their mutual trust. However, the PUs and CUs cooperate not in a gametheoretical sense, but interacts of relaying. Although the PDA may not converge to a stable equilibrium in a single-shot game, it does converge to an equilibrium in the repeated game as enforced by the threat of punishment in case of defection from cooperation.

\section{REFERENCES}

[1] X. Chen, H. Chen, and W. Meng, "Cooperative communications for cognitive radio networks: From theory to applications," IEEE Communications Surveys Tutorials, vol. 16, pp. 1180-1192, March 2014.

[2] H. Ogawa, D. Polifko, and S. Banba, "Millimeter-wave fiber optics systems for personal radio communication," IEEE Transactions on Microwave Theory and Techniques, vol. 40, pp. 2285-2293, Dec 1992.

[3] T. Komine, J. Lee, S. Haruyama, and M. Nakagawa, "Adaptive equalization system for visible light wireless communication utilizing multiple white led lighting equipment," IEEE Transactions on Wireless Communications, vol. 8, pp. 2892-2900, June 2009. 
4] R. Piesiewicz, C. Jansen, D. Mittleman, T. Kleine-Ostmann, M. Koch, and T. Kurner, "Scattering analysis for the modeling of thz communication systems," IEEE Transactions on Antennas and Propagation, vol. 55, pp. 3002-3009, Nov 2007.

[5] T. Yucek and H. Arslan, "A survey of spectrum sensing algorithms for cognitive radio applications," IEEE Communications Surveys Tutorials, vol. 11, pp. $116-130$, Quarter 2009.

[6] J. Mitola and G. Q. Maguire, "Cognitive radio: making software radios more personal," IEEE Personal Communications, vol. 6, pp. $13-18$, Aug 1999

[7] A. Goldsmith, S. Jafar, I. Maric, and S. Srinivasa, "Breaking spectrum gridlock with cognitive radios: An information theoretic perspective," Proceedings of the IEEE, pp. 894 -914, May 2009.

[8] S. Haykin, "Cognitive radio: brain-empowered wireless communications," IEEE Journal on Selected Areas in Communications, vol. 23, pp. $201-220$, Feb. 2005.

[9] F. C. Commission, "Spectrum policy task force," Rep. ET Docket no. 02-135, Nov 2002

[10] W. Zhang and K. Letaief, "Cooperative spectrum sensing with transmit and relay diversity in cognitive radio networks - [transaction letters]," IEEE Transactions on Wireless Communications, pp. $4761-4766$, December 2008

[11] G. Ganesan and Y. Li, "Cooperative spectrum sensing in cognitive radio, Part i: Two user networks," IEEE Transactions on Wireless Communications, vol. 6, pp. 2204 -2213, June 2007.

[12] A. Baker, S. Ghosh, A. Kumar, and M. Bayoumi, "Notice of Violation of IEEE Publication Principles LDPC decoder: A cognitive radio perspective for next generation (XG) communication," IEEE Circuits and Systems Magazine, vol. 7, pp. 24-37, March 2007.

[13] J. Jia, Q. Zhang, and X. S. Shen, "HC-MAC: A Hardware-Constrained Cognitive MAC for Efficient Spectrum Management," IEEE Journal on Selected Areas in Communications, vol. 26, pp. 106-117, Jan 2008.

[14] Z. Li, F. R. Yu, and M. Huang, "A Distributed Consensus-Based Cooperative Spectrum-Sensing Scheme in Cognitive Radios," IEEE Transactions on Vehicular Technology, vol. 59, pp. 383-393, Jan 2010.

[15] F. R. Yu, M. Huang, and H. Tang, "Biologically inspired consensusbased spectrum sensing in mobile Ad Hoc networks with cognitive radios," IEEE Network, vol. 24, pp. 26-30, May-June 2010.

[16] A. Attar, H. Tang, A. V. Vasilakos, F. R. Yu, and V. C. M. Leung, "A Survey of Security Challenges in Cognitive Radio Networks: Solutions and Future Research Directions," Proceedings of the IEEE, vol. 100, pp. 3172-3186, Dec 2012.

[17] Q. Guan, F. R. Yu, S. Jiang, and G. Wei, "Prediction-Based Topology Control and Routing in Cognitive Radio Mobile Ad Hoc Networks," IEEE Transactions on Vehicular Technology, vol. 59, pp. 4443-4452, Nov 2010.

[18] K. J. R. Liu, A. K. Sadek, W. Su and A. Kwasinski, Cooperative Communications and Networking. Cambridge University Press, New York, 2009.

[19] A. Sendonaris, E. Erkip, and B. Aazhang, "User cooperation diversity. Part I: System description," IEEE Transactions on Communications, vol. 51, pp. 1927 - 1938, Nov 2003.

[20] A. Sendonaris, E. Erkip, and B. Aazhang, "User cooperation diversity. Part II. Implementation aspects and performance analysis," IEEE Transactions on Communications, vol. 51, pp. 1939-1948, Nov 2003.

[21] K. Azarian, H. E. Gamal, and P. Schniter, "On the achievable diversity-multiplexing tradeoff in half-duplex cooperative channels," IEEE Transactions on Information Theory, vol. 51, pp. 4152-4172, Dec 2005.

[22] A. Bletsas, A. Khisti, D. Reed, and A. Lippman, "A simple cooperative diversity method based on network path selection," IEEE Journal on Selected Areas in Communications, vol. 24, pp. 659-672, March 2006.

[23] A. Host-Madsen and J. Zhang, "Capacity bounds and power allocation for wireless relay channels," IEEE Transactions on Information Theory, vol. 51, pp. 2020-2040, June 2005.

[24] T. Hunter and A. Nosratinia, "Diversity through coded cooperation," IEEE Transactions on Wireless Communications, , vol. 5, pp. 283-289, Feb 2006.

[25] B. Rankov and A. Wittneben, "Spectral efficient protocols for halfduplex fading relay channels," IEEE Journal on Selected Areas in Communications, vol. 25, pp. 379-389, February 2007.

[26] B. Rankov and A. Wittneben, "Achievable rate regions for the two-way relay channel," in 2006 IEEE International Symposium on Information Theory, pp. 1668-1672, July 2006.

[27] Y. Fan, C. Wang, J. Thompson, and H. Poor, "Recovering multiplexing loss through successive relaying using repetition coding," IEEE Transactions on Wireless Communications, vol. 6, pp. 4484-4493, December 2007.
[28] Y. Han, A. Pandharipande, and S. Ting, "Cooperative decode-andforward relaying for secondary spectrum access," IEEE Transactions on Wireless Communications, vol. 8, pp. 4945 -4950, October 2009.

[29] Q. Zhang, J. Jia, and J. Zhang, "Cooperative relay to improve diversity in cognitive radio networks," IEEE Communications Magazine, vol. 47, pp. 111-117, February 2009.

[30] O. Simeone, Y. Bar-Ness, and U. Spagnolini, "Stable throughput of cognitive radios with and without relaying capability," IEEE Transactions on Communications, vol. 55, pp. 2351-2360, Dec 2007.

[31] Y. Chen, G. Yu, Z. Zhang, H. Chen, and P. Qiu, "On cognitive radio networks with opportunistic power control strategies in fading channels," IEEE Transactions on Wireless Communications, vol. 7 , pp. 2752-2761, July 2008.

[32] Y. Zou, J. Zhu, B. Zheng, and Y. Yao, "An adaptive cooperation diversity scheme with best-relay selection in cognitive radio networks," IEEE Transactions on Signal Processing, vol. 58, pp. 5438-5445, Oct 2010.

[33] Y. Zou, Y. Yao, and B. Zheng, "Cognitive transmissions with multiple relays in cognitive radio networks," IEEE Transactions on Wireless Communications, vol. 10, pp. 648-659, February 2011.

[34] L. Li, X. Zhou, H. Xu, G. Li, D. Wang, and A. Soong, "Simplified relay selection and power allocation in cooperative cognitive radio systems," IEEE Transactions on Wireless Communications, vol. 10, pp. 33-36, January 2011.

[35] P. Ubaidulla and S. Aissa, "Optimal relay selection and power allocation for cognitive two-way relaying networks," IEEE Wireless Communications Letters, vol. 1, pp. 225-228, June 2012.

[36] G. Zhao, C. Yang, G. Li, D. Li, and A. Soong, "Power and channel allocation for cooperative relay in cognitive radio networks," IEEE Journal of Selected Topics in Signal Processing, vol. 5, pp. 151-159, Feb 2011.

[37] R. Zhang, Y. Liang, and S. Cui, "Dynamic resource allocation in cognitive radio networks," IEEE Signal Processing Magazine, vol. 27, pp. 102-114, May 2010.

[38] J. Liang and J. Chen, "Resource allocation in cognitive radio relay networks," IEEE Journal on Selected Areas in Communications, vol. 31, pp. 476-488, March 2013.

[39] Y. Tachwali, B. Lo, I. Akyildiz, and R. Agusti, "Multiuser resource allocation optimization using bandwidth-power product in cognitive radio networks," IEEE Journal on Selected Areas in Communications, vol. 31, pp. 451-463, March 2013.

[40] Q. Li, S. H. Ting, A. Pandharipande, and Y. Han, "Cognitive spectrum sharing with two-way relaying systems," IEEE Transactions on Vehicular Technology, vol. 60, pp. 1233-1240, March 2011.

[41] $\mathrm{H}$. $\mathrm{Mu}$ and J. Tugnait, "Interference alignment-like precoder design in multi-pair two-way relay cognitive radio networks," in IEEE Global Communications Conference (GLOBECOM), pp. 1471-1476, Dec 2012.

[42] Q. Zhang, S. Kota, V. Lau, W. Su, and A. Kwasinski, "Introduction to the issue on cooperative communication and signal processing in cognitive radio systems," IEEE Journal of Selected Topics in Signal Processing, vol. 5, pp. 1-4, Feb 2011.

[43] N. Devroye, P. Mitran, and V. Tarokh, "Achievable rates in cognitive radio channels," IEEE Transactions on Information Theory, vol. 52, pp. 1813 - 1827, May 2006.

[44] W.F. Su and J.D. Matyjas and S. Batalama, "Active cooperation between primary users and cognitive radio users in cognitive ad-hoc networks," in 2010 IEEE International Conference on Acoustics Speech and Signal Processing (ICASSP), pp. 3174 -3177, March 2010.

[45] W. Su, J. Matyjas, and S. Batalama, "Active cooperation between primary users and cognitive radio users in heterogeneous ad-hoc networks," IEEE Transactions on Signal Processing, , vol. 60, pp. 1796 -1805 , April 2012.

[46] B. Cao, Q. Zhang, J. W. Mark, L. Cai, and H. Poor, "Toward efficient radio spectrum utilization: user cooperation in cognitive radio networking," IEEE Network, vol. 26, pp. 46-52, July 2012.

[47] O. Simeone, J. Gambini, Y. B. Ness, and U. Spagnolini, "Cooperation and cognitive radio," in IEEE International Conference on Communications (ICC '07), pp. 6511 -6515, June 2007.

[48] S. Mallick, R. Devarajan, R. Loodaricheh, and V. Bhargava, "Robust resource optimization for cooperative cognitive radio networks with imperfect CSI," IEEE Transactions on Wireless Communications, vol. 14, pp. 907-920, Feb 2015.

[49] J. Hayes, "Adaptive Feedback Communications," IEEE Transactions on Communication Technology, vol. 16, pp. 29-34, February 1968.

[50] Y. Zeng and Y.-C. Liang, "Spectrum-sensing algorithms for cognitive radio based on statistical covariances," IEEE Transactions on Vehicular Technology, vol. 58, pp. 1804 -1815, May 2009. 
[51] S. Haykin, D. Thomson, and J. Reed, "Spectrum sensing for cognitive radio," Proceedings of the IEEE, pp. 849 -877, May 2009.

[52] Z. Tian and G. B. Giannakis, "A wavelet approach to wideband spectrum sensing for cognitive radios," in 1st International Conference on Cognitive Radio Oriented Wireless Networks and Communications, pp. 1 -5, June 2006.

[53] Z. Quan, S. Cui, A. Sayed, and H. Poor, "Optimal multiband joint detection for spectrum sensing in cognitive radio networks," IEEE Transactions on Signal Processing, pp. 1128 -1140, March 2009.

[54] R. Niu, B. Chen, and P. Varshney, "Fusion of decisions transmitted over rayleigh fading channels in wireless sensor networks," IEEE Transactions on Signal Processing, pp. 1018 - 1027, March 2006.

[55] I. Akyildiz, W.-Y. Lee, M. Vuran, and S. Mohanty, "A survey on spectrum management in cognitive radio networks," IEEE Communications Magazine, vol. 46, pp. 40-48, April 2008.

[56] C. He, Z. Feng, Q. Zhang, Z. Zhang, and H. Xiao, "A joint relay selection, spectrum allocation and rate control scheme in relay-assisted cognitive radio system," in IEEE 72nd Vehicular Technology Conference Fall (VTC 2010-Fall), pp. 1-5, Sept 2010.

[57] H. Wang, L. Gao, X. Gan, X. Wang, and E. Hossain, "Cooperative spectrum sharing in cognitive radio networks: A game-theoretic approach," in 2010 IEEE International Conference on Communications (ICC), pp. 1-5, May 2010.

[58] X. Qiao, Z. Tan, S. Xu, and J. Li, "Combined power allocation in cognitive radio-based relay-assisted networks," in 2010 IEEE International Conference on Communications Workshops (ICC), pp. 1-5, May 2010.

[59] B. Wang, Z. Han, and K. Liu, "Distributed relay selection and power control for multiuser cooperative communication networks using stackelberg game," IEEE Transactions on Mobile Computing, vol. 8, pp. 975-990, July 2009.

[60] Y. Xiao, G. Bi, and D. Niyato, "Game theoretic analysis for spectrum sharing with multi-hop relaying," IEEE Transactions on Wireless Communications, vol. 10, pp. 1527-1537, May 2011.

[61] W. Liang, S. Ng, J. Feng, and L. Hanzo, "Pragmatic distributed algorithm for spectral access in cooperative cognitive radio networks," IEEE Transactions on Communications, vol. 62, pp. 1188-1200, April 2014.

[62] L. Gao, L. Duan, and J. Huan, "Two-sided matching based cooperative spectrum sharing," IEEE Transactions on Mobile Computing, vol. PP, no. 99 , pp. 1-1, 2016

[63] D. Li, Y. Xu, X. Wang, and M. Guizani, "Coalitional game theoretic approach for secondary spectrum access in cooperative cognitive radio networks," IEEE Transactions on Wireless Communications, vol. 10, no. 3, pp. 844-856, 2011.

[64] B. Di, S. Bayat, L. Song, and Y. Li, "Radio Resource Allocation for Downlink Non-Orthogonal Multiple Access (NOMA) Networks Using Matching Theory," in 2015 IEEE Global Communications Conference (GLOBECOM), pp. 1-6, Dec 2015.

[65] R. Ruby, V. C. M. Leung, and D. G. Michelson, "Centralized and Game Theoretical Solutions of Joint Source and Relay Power Allocation for AF Relay Based Network," IEEE Transactions on Communications, vol. 63, pp. 2848-2863, Aug 2015.

[66] G. Scutari, D. Palomar, and S. Barbarossa, "Optimal linear precoding strategies for wideband non-cooperative systems based on game theory part II: Algorithms," IEEE Transactions on Signal Processing, vol. 56, pp. 1250-1267, March 2008.

[67] Z. Han, Z. Ji, and K. Liu, "Non-cooperative resource competition game by virtual referee in multi-cell OFDMA networks," IEEE Journal on Selected Areas in Communications, vol. 25, pp. 1079-1090, August 2007.

[68] L. Zhao, J. Zhang, and H. Zhang, "Using incompletely cooperative game theory in wireless mesh networks," IEEE Network, vol. 22, pp. 39-44, Jan 2008.

[69] E. Larsson and E. Jorswieck, "Competition versus cooperation on the miso interference channel," IEEE Journal on Selected Areas in Communications, vol. 26, pp. 1059-1069, September 2008.

[70] L. Giupponi and C. Ibars, "Distributed cooperation in cognitive radio networks: Overlay versus underlay paradigm," in IEEE 69th Vehicular Technology Conference, 2009. VTC Spring 2009., pp. 1-6, April 2009.

[71] C. Jiang, L. Duan, and J. Huang, "Optimal Pricing and Admission Control for Heterogeneous Secondary Users," IEEE Transactions on Wireless Communications, vol. 15, pp. 5218-5230, Aug 2016.

[72] X. Chen and J. Huang, "Database-assisted distributed spectrum sharing," IEEE Journal on Selected Areas in Communications, vol. 31, pp. 2349-2361, November 2013.

[73] M. Naeem, A. Anpalagan, M. Jaseemuddin, and D. Lee, "Resource allocation techniques in cooperative cognitive radio networks," IEEE Communications Surveys Tutorials, vol. 16, pp. 729-744, Second 2014.
[74] G. Bansal, O. Duval, and F. Gagnon, "Joint overlay and underlay power allocation scheme for ofdm-based cognitive radio systems," in IEEE 71st Vehicular Technology Conference (VTC 2010-Spring), pp. 1-5, May 2010.

[75] A. Marques, L. Lopez-Ramos, G. Giannakis, and J. Ramos, "Resource allocation for interweave and underlay crs under probabilityof-interference constraints," IEEE Journal on Selected Areas in Communications, vol. 30, pp. 1922-1933, November 2012.

[76] Q. Zhao and B. Sadler, "A survey of dynamic spectrum access," IEEE Signal Processing Magazine, vol. 24, pp. 79-89, May 2007.

[77] R. Manna, R. H. Louie, Y. Li, and B. Vucetic, "Cooperative spectrum sharing in cognitive radio networks with multiple antennas," IEEE Transactions on Signal Processing, vol. 59, pp. 5509-5522, Nov 2011.

[78] J. N. L. D. N. C. Tse and G. W. Wornell, "Cooperative diversity in wireless networks: Efficient protocols and outage behavior," IEEE Transactions on Information Theory, vol. 50, pp. 3062-3080, Dec 2004.

[79] K. Wang, L. Chen, and Q. Liu, "Opportunistic spectrum access by exploiting primary user feedbacks in underlay cognitive radio systems: An optimality analysis," IEEE Journal of Selected Topics in Signal Processing, vol. 7, pp. 869-882, Oct 2013.

[80] M. G. Khoshkholgh, K. Navaie, and H. Yanikomeroglu, "Interference management in underlay spectrum sharing using indirect power control signalling," IEEE Transactions on Wireless Communications, vol. 12, pp. 3264-3277, July 2013.

[81] G. Chen, Z. Tian, Y. Gong, and J. Chambers, "Decode-and-forward buffer-aided relay selection in cognitive relay networks," IEEE Transactions on Vehicular Technology, vol. 63, pp. 4723-4728, Nov 2014.

[82] E. E. B. Olivo, D. P. M. Osorio, D. B. da Costa, and J. C. S. S. Filho, "Outage performance of spectrally efficient schemes for multiuser cognitive relaying networks with underlay spectrum sharing," IEEE Transactions on Wireless Communications, vol. 13, pp. 6629-6642, Dec 2014

[83] M. Rasti, M. Hasan, L. B. Le, and E. Hossain, "Distributed uplink power control for multi-cell cognitive radio networks," IEEE Transactions on Communications, vol. 63, pp. 628-642, March 2015.

[84] K. Huang, V. K. N. Lau, and Y. Chen, "Spectrum sharing between cellular and mobile ad hoc networks: transmission-capacity trade-off," IEEE Journal on Selected Areas in Communications, vol. 27, pp. 12561267, September 2009.

[85] G. Gur, S. Bayhan, and F. Alagoz, "Cognitive femtocell networks: an overlay architecture for localized dynamic spectrum access [dynamic spectrum management]," IEEE Wireless Communications, vol. 17, pp. 62-70, August 2010.

[86] X. Lin, J. G. Andrews, and A. Ghosh, "Spectrum sharing for deviceto-device communication in cellular networks," IEEE Transactions on Wireless Communications, vol. 13, pp. 6727-6740, Dec 2014.

[87] T. Kwon and J. M. Cioffi, "Spatial spectrum sharing for heterogeneous simo networks," IEEE Transactions on Vehicular Technology, vol. 63, pp. 688-702, Feb 2014.

[88] A. A. El-Sherif and A. Mohamed, "Joint routing and resource allocation for delay minimization in cognitive radio based mesh networks," IEEE Transactions on Wireless Communications, vol. 13, pp. 186-197, January 2014.

[89] T. K. Kim, H. M. Kim, M. G. Song, and G. H. Im, "Improved spectrumsharing protocol for cognitive radio networks with multiuser cooperation," IEEE Transactions on Communications, vol. 63, pp. 1121-1135, April 2015.

[90] Z. Han, D. Niyato, W. Saad, T. Basar, and A. Hjorungnes, Game Theory in Wireless and Communication Networks: Theory, Models and Applications. Cambridge University Press , 2012.

[91] K. Akkarajitsakul, E. Hossain, D. Niyato, and D. I. Kim, "Game theoretic approaches for multiple access in wireless networks: A survey," IEEE Communications Surveys Tutorials, vol. 13, pp. 372395, Third 2011.

[92] L. Gavrilovska, V. Atanasovski, I. Macaluso, and L. DaSilva, "Learning and reasoning in cognitive radio networks," IEEE Communications Surveys Tutorials, vol. 15, pp. 1761-1777, April 2013.

[93] Y. Xu, A. Anpalagan, Q. Wu, L. Shen, Z. Gao, and J. Wang, "Decisiontheoretic distributed channel selection for opportunistic spectrum access: Strategies, challenges and solutions," IEEE Communications Surveys Tutorials, vol. 15, pp. 1689-1713, April 2013.

[94] D. Niyato, E. Hossain, and Z. Han, "Dynamic spectrum access in ieee 802.22- based cognitive wireless networks: a game theoretic model for competitive spectrum bidding and pricing," IEEE Wireless Communications, vol. 16, pp. 16-23, April 2009.

[95] J. Huang, Z. Han, M. Chiang, and H. V. Poor, "Auction-based resource allocation for cooperative communications," IEEE Journal on Selected Areas in Communications, vol. 26, pp. 1226-1237, September 2008. 
[96] X. Wang, Z. Li, P. Xu, Y. Xu, X. Gao, and H. H. Chen, "Spectrum sharing in cognitive radio networks 2014; an auction-based approach," IEEE Transactions on Systems, Man, and Cybernetics, Part B (Cybernetics), vol. 40, pp. 587-596, June 2010.

[97] N. Wang and T. A. Gulliver, "Distributed queue-aware relay node selection for cooperative wireless networks via vickrey auction game," IEEE Wireless Communications Letters, vol. 4, pp. 257-260, June 2015.

[98] Y. Wu, B. Wang, K. J. R. Liu, and T. C. Clancy, "Repeated open spectrum sharing game with cheat-proof strategies," IEEE Transactions on Wireless Communications, vol. 8, pp. 1922-1933, April 2009.

[99] P. Zhou, Y. Chang, and J. Copeland, "Reinforcement learning for repeated power control game in cognitive radio networks," IEEE Journal on Selected Areas in Communications, vol. 30, pp. 54-69, January 2012.

[100] Y. Xiao, J. Park, and M. van der Schaar, "Repeated games with intervention: Theory and applications in communications," IEEE Transactions on Communications, vol. 60, pp. 3123-3132, October 2012.

[101] S. Hamouda, I. B. Chaabane, and S. Tabbane, "Cooperative bandwidth sharing for relaying in lte-advanced using game theory," IEEE Transactions on Vehicular Technology, vol. 64, pp. 2306-2317, June 2015.

[102] M. Haddad, S. Elayoubi, E. Altman, and Z. Altman, "A hybrid approach for radio resource management in heterogeneous cognitive networks," IEEE Journal on Selected Areas in Communications, vol. 29, pp. 831-842, April 2011.

[103] G. Nan, Z. Mao, M. Yu, M. Li, H. Wang, and Y. Zhang, "Stackelberg game for bandwidth allocation in cloud-based wireless live-streaming social networks," IEEE Systems Journal, vol. 8, pp. 256-267, March 2014.

[104] K. Zhu and E. Hossain, "Joint mode selection and spectrum partitioning for device-to-device communication: A dynamic stackelberg game," IEEE Transactions on Wireless Communications, vol. 14, pp. 14061420, March 2015.

[105] C. Yi and J. Cai, "Two-stage spectrum sharing with combinatorial auction and stackelberg game in recall-based cognitive radio networks," IEEE Transactions on Communications, vol. 62, pp. 3740-3752, Nov 2014.

[106] M. H. Cheung, V. W. S. Wong, and R. Schober, "Sinr-based random access for cognitive radio: Distributed algorithm and coalitional game," IEEE Transactions on Wireless Communications, vol. 10, pp. 38873897, November 2011.

[107] R. Mochaourab and E. A. Jorswieck, "Coalitional games in miso interference channels: Epsilon-core and coalition structure stable set," IEEE Transactions on Signal Processing, vol. 62, pp. 6507-6520, Dec 2014.

[108] A. Attar, M. Nakhai, and A. Aghvami, "Cognitive radio game for secondary spectrum access problem," IEEE Transactions on Wireless Communications, vol. 8, pp. 2121-2131, April 2009.

[109] G. Zhang, K. Yang, and H. H. Chen, "Resource allocation for wireless cooperative networks: a unified cooperative bargaining game theoretic framework," IEEE Wireless Communications, vol. 19, pp. 38-43, April 2012.

[110] X. Liu and E. Erkip, "A game-theoretic view of the interference channel: Impact of coordination and bargaining," IEEE Transactions on Information Theory, vol. 57, pp. 2805-2820, May 2011.

[111] H. Zhang, C. Jiang, N. C. Beaulieu, X. Chu, X. Wang, and T. Q. S. Quek, "Resource allocation for cognitive small cell networks: A cooperative bargaining game theoretic approach," IEEE Transactions on Wireless Communications, vol. 14, pp. 3481-3493, June 2015.

[112] D. Niyato and E. Hossain, "Competitive pricing for spectrum sharing in cognitive radio networks: Dynamic game, inefficiency of nash equilibrium, and collusion," IEEE Journal on Selected Areas in Communications, vol. 26, pp. 192-202, Jan 2008.

[113] M. L. Treust and S. Lasaulce, "A repeated game formulation of energyefficient decentralized power control," IEEE Transactions on Wireless Communications, vol. 9, pp. 2860-2869, September 2010.

[114] J. Torrance and L. Hanzo, "Optimisation of switching levels for adaptive modulation in slow Rayleigh fading," Electronics Letters, vol. 32, pp. 1167-1169, Jun 1996.

[115] A. Goldsmith and S.-G. Chua, "Adaptive coded modulation for fading channels," IEEE Transactions on Communications, vol. 46, pp. 595602, May 1998.

[116] S. X. Ng, C. Wong, and L. Hanzo, "Burst-by-burst adaptive decision feedback equalized TCM, TTCM, BICM and BICM-ID," in IEEE International Conference on Communications (ICC 2001), vol. 10, pp. 3031-3035, 2001.

[117] L. Hanzo and S. X. Ng and T. Keller and W. Webb, Quadrature Amplitude Modulation:From Basics to Adaptive Trellis-Coded, Turbo-
Equalised and Space-Time Coded OFDM, CDMA and MC-CDMA Systems. Wiley-IEEE Press, 2004.

[118] L. hanzo and C. H. Wong and and M. S. Yee, Adaptive Wireless Transceivers: Turbo-Coded, Turbo-Equalized and Space-Time Coded TDMA, CDMA and OFDM Systems. John Wiley Sons, Ltd, 2002.

[119] I. Djordjevic, "Adaptive Modulation and Coding for Free-Space Optical Channels," IEEE/OSA Journal of Optical Communications and Networking, vol. 2, pp. 221-229, May 2010.

[120] J. Jiang, J. Thompson, and H. Sun, "A Singular-Value-Based Adaptive Modulation and Cooperation Scheme for Virtual-MIMO Systems," IEEE Transactions on Vehicular Technology, vol. 60, pp. 2495-2504, July 2011.

[121] G. Piro, L. Grieco, G. Boggia, F. Capozzi, and P. Camarda, "Simulating LTE Cellular Systems: An Open-Source Framework," IEEE Transactions on Vehicular Technology, vol. 60, pp. 498-513, Feb 2011.

[122] P. Li, H. Zhang, B. Zhao, and S. Rangarajan, "Scalable Video Multicast With Adaptive Modulation and Coding in Broadband Wireless Data Systems," IEEE/ACM Transactions on Networking, vol. 20, pp. 57-68, Feb 2012.

[123] N. Mastronarde and M. van der Schaar, "Joint Physical-Layer and System-Level Power Management for Delay-Sensitive Wireless Communications," IEEE Transactions on Mobile Computing, vol. 12, pp. 694-709, April 2013.

[124] J. Yoon, H. Zhang, S. Banerjee, and S. Rangarajan, "Video Multicast With Joint Resource Allocation and Adaptive Modulation and Coding in 4G Networks," IEEE/ACM Transactions on Networking, vol. 22, pp. 1531-1544, Oct 2014.

[125] L. Wan, H. Zhou, X. Xu, Y. Huang, S. Zhou, Z. Shi, and J.-H. Cui, "Adaptive modulation and coding for underwater acoustic ofdm," IEEE Journal of Oceanic Engineering, vol. 40, pp. 327-336, April 2015.

[126] W. Liang, S. Ng, and L. Hanzo, "Adaptive turbo trellis coded modulation aided cooperative cognitive radio," in 2012 IEEE Wireless Communications and Networking Conference (WCNC), pp. 2362 2366, April 2012.

[127] W. Liang, S. X. Ng, and L. Hanzo, "Cooperative communication between cognitive and primary users," IET Communications, vol. 7, no. 17, pp. 1982-1992, 2013.

[128] W. Liang, S. X. Ng, J. Feng, and L. Hanzo, "Pragmatic distributed algorithm for spectral access in cooperative cognitive radio networks," IEEE Transactions on Communications, vol. 62, pp. 1188-1200, April 2014.

[129] H. Ochiai, P. Mitran, and V. Tarokh, "Design and analysis of collaborative diversity protocols for wireless sensor networks," in IEEE 60th Vehicular Technology Conference, 2004 (VTC2004-Fall), vol. 7, pp. 4645 - 4649 Vol. 7, Sept. 2004.

[130] G. Li, Z. Xu, C. Xiong, C. Yang, S. Zhang, Y. Chen, and S. Xu, "Energy-efficient wireless communications: tutorial, survey, and open issues," IEEE Wireless Communications, vol. 18, pp. 28 -35, December 2011.

[131] S. Ng and L. Hanzo, "On the MIMO channel capacity of multidimensional signal sets," IEEE Transactions on Vehicular Technology, vol. 55, pp. 528 - 536, March 2006.

[132] S. Hu, Z. Ding, Q. Ni, and Y. Yuan, "Beamforming optimization for full-duplex cooperative cognitive radio networks," in 2016 IEEE 17th International Workshop on Signal Processing Advances in Wireless Communications (SPAWC), pp. 1-5, July 2016.

[133] Y. Deng, K. J. Kim, T. Q. Duong, M. Elkashlan, G. K. Karagiannidis, and A. Nallanathan, "Full-duplex spectrum sharing in cooperative single carrier systems," IEEE Transactions on Cognitive Communications and Networking, vol. 2, pp. 68-82, March 2016

[134] E. E. B. Olivo, D. P. M. Osorio, H. Alves, J. C. S. S. Filho, and M. Latva-aho, "An adaptive transmission scheme for cognitive decode-and-forward relaying networks: Half duplex, full duplex, or no cooperation," IEEE Transactions on Wireless Communications, vol. 15, pp. 5586-5602, Aug 2016.

[135] C. Zhai, J. Liu, and L. Zheng, "Cooperative spectrum sharing with wireless energy harvesting in cognitive radio networks," IEEE Transactions on Vehicular Technology, vol. 65, pp. 5303-5316, July 2016.

[136] S. Bayat, R. Louie, Y. Li, and B. Vucetic, "Cognitive radio relay networks with multiple primary and secondary users: Distributed stable matching algorithms for spectrum access," in 2011 IEEE International Conference on Communications (ICC), pp. 1 -6, June 2011.

[137] S. Bayat, R. Louie, Z. Han, Y. Li, and B. Vucetic, "Distributed stable matching algorithm for physical layer security with multiple source-destination pairs and jammer nodes," in 2012 IEEE Wireless Communications and Networking Conference (WCNC), pp. 2688 2693, April 2012. 
[138] L. Jayasinghe, N. Rajatheva, and M. Latva-aho, "Optimal power allocation for pnc relay based communications in cognitive radio," in 2011 IEEE International Conference on Communications (ICC), pp. 15, June 2011.

[139] L. Zhang, Y.-C. Liang, and Y. Xin, "Joint beamforming and power allocation for multiple access channels in cognitive radio networks," IEEE Journal on Selected Areas in Communications, vol. 26, pp. 3851, Jan 2008.

[140] M. Tao and Y. Liu, "Spectrum leasing and cooperative resource allocation in cognitive ofdma networks," Journal of Communications and Networks, vol. 15, pp. 102-110, Feb 2013.

[141] Y. Pei and Y. Liang, "Cooperative spectrum sharing with bidirectional secondary transmissions," IEEE Transactions on Vehicular Technology, vol. 64, pp. 108-117, Jan 2015.

[142] M. Shamaian, S. H. Lee, S. Vishwanath, and H. Vikalo, "Distributed algorithms for spectrum access in cognitive radio relay networks," IEEE Journal on Selected Areas in Communications, vol. 30, pp. $1947-1957$, November 2012.

[143] J. Tadrous, A. Sultan, M. Nafie, and A. El-Keyi, "Power control for constrained throughput maximization in spectrum shared networks," in IEEE Global Telecommunications Conference (GLOBECOM 2010), pp. 1-6, Dec 2010 .

[144] H. Zhou, B. Liu, Y. Liu, N. Zhang, L. Gui, Y. Li, X. Shen, and $\mathrm{Q}$. Yu, "A cooperative matching approach for resource management in dynamic spectrum access networks," IEEE Transactions on Wireless Communications, vol. 13, pp. 1047-1057, February 2014.

[145] P. Lin, J. Jia, Q. Zhang, and M. Hamdi, "Dynamic spectrum sharing with multiple primary and secondary users," IEEE Transactions on Vehicular Technology, vol. 60, pp. 1756 -1765, may 2011.

[146] S. Gmira, A. Kobbane, E. Sabir, and J. Ben-othman, "A game theoretic approach for an hybrid overlay-underlay spectrum access mode," in 2016 IEEE International Conference on Communications (ICC), pp. 16, May 2016.

[147] S. Vassaki, M. I. Poulakis, A. D. Panagopoulos, and P. Constantinou, "An auction-based mechanism for spectrum leasing in overlay cognitive radio networks," in 2013 IEEE 24th Annual International Symposium on Personal, Indoor, and Mobile Radio Communications (PIMRC), pp. 2733-2737, Sept 2013

[148] J. Zou, H. Xiong, D. Wang, and C. W. Chen, "Optimal power allocation for hybrid overlay/underlay spectrum sharing in multiband cognitive radio networks," IEEE Transactions on Vehicular Technology, vol. 62, pp. 1827-1837, May 2013.

[149] F. Ouyang, J. Ge, and J. Hou, "Cooperative waiting-time reduction for cognitive radio networks using stackelberg game," IET Communications, vol. 8, no. 17, pp. 3072-3080, 2014.

[150] O. Simeone, I. Stanojev, S. Savazzi, Y. Bar-Ness, U. Spagnolini, and R. Pickholtz, "Spectrum leasing to cooperating secondary ad hoc networks," IEEE Journal on Selected Areas in Communications, vol. 26, no. 1, pp. 203-213, 2008.

[151] X. Wang, K. Ma, Z. L. Q. Han, and X. Guan, "Pricing-based spectrum leasing in cognitive radio networks," IET Networks, vol. 1, no. 3, pp. 116-125, 2012

[152] X. Hao, M. H. Cheung, V. W. S. Wong, and V. C. M. Leung, "A stackelberg game for cooperative transmission and random access in cognitive radio networks," in 2011 IEEE 22nd International Symposium on Personal, Indoor and Mobile Radio Communications, pp. 411-416, Sept 2011.

[153] L. S. Pillutla and V. Krishnamurthy, "Game theoretic rate adaptation for spectrum-overlay cognitive radio networks," in IEEE GLOBECOM 2008 - 2008 IEEE Global Telecommunications Conference, pp. 1-5, Nov 2008.

[154] D. Gusfield and R. W. Irving, The Stable Marriage Problem: Structure and Algorithms. The MIT Press , 1989.

[155] A. Roth and M. Sotomanyor, Two sided matching: a study in gametheoretic modeling and analysis, 1st ed. Cambridge University Press, 1989.

[156] Y. Gu, W. Saad, M. Bennis, M. Debbah, and Z. Han, "Matching theory for future wireless networks: fundamentals and applications," IEEE Communications Magazine, vol. 53, pp. 52-59, May 2015.

[157] L. Gao, L. Duan, and J. Huang, "Two-sided matching based cooperative spectrum sharing," IEEE Transactions on Mobile Computing, vol. PP, no. 99 , pp. 1-1, 2016.

[158] B. Di, S. Bayat, L. Song, and Y. Li, "Radio Resource Allocation for Downlink Non-Orthogonal Multiple Access (NOMA) Networks Using Matching Theory," in 2015 IEEE Global Communications Conference (GLOBECOM), pp. 1-6, Dec 2015.
[159] D. P. Bertsekas, "A distributed asynchronous relaxation algorithm for the assignment problem," in 24th IEEE Conference on Decision and Control,, vol. 24, pp. $1703-1704$, Dec. 1985.

[160] C. An, L. Zhang, and W. Liu, "A spectrum allocation algorithm based on matching game,' in WiCom '09. 5th International Conference on Wireless Communications, Networking and Mobile Computing, pp. 1 -3 , Sept. 2009.

[161] V. Asghari and S. Aissa, "Resource management in spectrum-sharing cognitive radio broadcast channels: Adaptive time and power allocation," IEEE Transactions on Communications, vol. 59, no. 5, pp. 14461457, 2011.

[162] S. El-Bessi, S. Hamouda, and S. Tabbane, "An efficient cooperative spectrum access in cognitive radio networks via coalitional game," in 2013 IEEE 78th Vehicular Technology Conference (VTC Fall), pp. 1-5, Sept 2013.

[163] B. Ma, M. H. Cheung, V. W. S. Wong, and J. Huang, "Hybrid overlay/underlay cognitive femtocell networks: A game theoretic approach," IEEE Transactions on Wireless Communications, vol. 14, pp. 32593270, June 2015.

[164] M. J. Osborne, An Introduction to Game Theory. Oxford University Press, 2004.

[165] R. J. Aumann and M. Maschler, Repeated games with incomplete information. The MIT Press, 1995.

[166] R.B. Myerson, Game Theory Analysis of Conflict. Harvard University Press, 1997.

[167] W. Saad, Z. Han, M. Debbah, A. Hjorungnes, and T. Basar, "Coalitional game theory for communication networks," IEEE Signal Processing Magazine, vol. 26, no. 5, pp. 77-97, 2009.

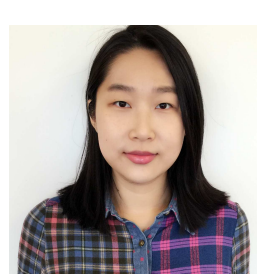

Wei Liang received her M.Sc. and Ph.D. degree in wireless communication at University of Southampton, Southampton, U.K in 2010 and 2015, respectively. She is a Postdoctoral Research Fellow in Lancaster University now. Her research interests include adaptive coded modulation, network coding, matching theory, game theory, cooperative communication, cognitive radio network and Non-orthogonal multiple access scheme.

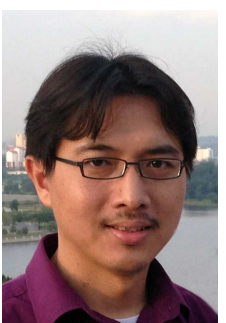

Dr Soon Xin Ng (S'99-M'03-SM'08) received the B.Eng. degree (First class) in electronic engineering and the Ph.D. degree in telecommunications from the University of Southampton, Southampton, U.K., in 1999 and 2002, respectively. From 2003 to 2006, he was a postdoctoral research fellow working on collaborative European research projects known as SCOUT, NEWCOM and PHOENIX. Since August 2006, he has been a member of academic staff in the School of Electronics and Computer Science, University of Southampton. He is involved in the OPTIMIX and CONCERTO European projects as well as the IU-ATC and UC4G projects. He is currently an Associate Professor in telecommunications at the University of Southampton.

His research interests include adaptive coded modulation, coded modulation, channel coding, space-time coding, joint source and channel coding, iterative detection, OFDM, MIMO, cooperative communications, distributed coding, quantum error correction codes and joint wireless-and-optical-fibre communications. He has published over 180 papers and co-authored two John Wiley/IEEE Press books in this field. He is a Senior Member of the IEEE, a Chartered Engineer and a Fellow of the Higher Education Academy in the UK. 


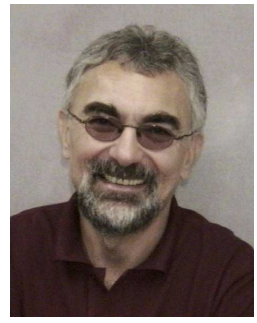

Lajos Hanzo (http://www-mobile.ecs.soton.ac.uk) FREng, FIEEE, FIET, Fellow of EURASIP, DSc received his degree in electronics in 1976 and his doctorate in 1983. In 2009 he was awarded an honorary doctorate by the Technical University of Budapest, while in 2015 by the University of Edinburgh. During his 40-year career in telecommunications he has held various research and academic posts in Hungary, Germany and the UK. Since 1986 he has been with the School of Electronics and Computer Science, University of Southampton, UK, where he holds the chair in telecommunications. He has successfully supervised 100+ PhD students, co-authored 18 John Wiley/IEEE Press books on mobile radio communications totalling in excess of 10000 pages, published 1650 research entries at IEEE Xplore, acted both as TPC and General Chair of IEEE conferences, presented keynote lectures and has been awarded a number of distinctions. Currently he is directing a 60-strong academic research team, working on a range of research projects in the field of wireless multimedia communications sponsored by industry, the Engineering and Physical Sciences Research Council (EPSRC) UK, the European Research Council's Advanced Fellow Grant and the Royal Society's Wolfson Research Merit Award. He is an enthusiastic supporter of industrial and academic liaison and he offers a range of industrial courses. He is also a Governor of the IEEE VTS. During 2008 - 2012 he was the Editor-in-Chief of the IEEE Press and a Chaired Professor also at Tsinghua University, Beijing. His research is funded by the European Research Council's Senior Research Fellow Grant. For further information on research in progress and associated publications please refer to http://www-mobile.ecs.soton.ac.uk Lajos has 30 000+ citations. 